\title{
NOTAS SOBRE LA POLITICA MONETARIA RECIENTE DE BRASIL
}

\author{
Affonso Celso Pastore*
}

\section{INTRODUCCIÓN}

El PRESEnte trabajo tiene tres objetivos. En primer lugar, se trata de construir un modelo para medir el impacto a corto plazo y al ajuste dinámico de la tasa de inflación debidos a cambios en la oferta monetaria, la tasa de salarios y al tipo de cambio.

Se ha demostrado que la tasa de inflación es una función de las tasas de cambio actuales y pasadas de la oferta monetaria, la tasa de salarios y el tipo de cambio, y que los retrasos en la respuesta del nivel de precios son prolongados. Por lo tanto, se tratará de medir la eficiencia de la política monetaria, identificar los instrumentos usados para reducir gradualmente la tasa de crecimiento de la oferta monetaria, concediendo atención especial al comportamiento del impuesto inflacionario durante el proceso de ajuste. Por último, se tratará de explicar el comportamiento de la oferta de préstamos reales del sistema bancario al sector privado y mostrar cómo fue posible hacer compatible la reducción gradual de la tasa de cambio de la oferta monetaria con el aumento continuo en la existencia real de los préstamos bancarios.

\section{DiNÁMICA DE LA INFLACIÓN}

Al contrario de algunas sugerencias recientes de que el nivel de precios podría depender muy poco a casi nada del comportamiento de la oferta monetaria, el presente modelo sigue la tradición de la teoría monetaria cuantitativa; ${ }^{1}$ se parte de la hipótesis de que la demanda monetaria a largo plazo es una función estable del ingreso real y el costo de retener el dinero, cuyo componente dominante es la tasa esperada de inflación.

$$
m_{t}^{d}=-\alpha E_{t}+\beta y_{t}
$$

* Agradezco a L. Taylor, M.A. Broda y R.D. Almonacid sus valiosas sugerencias, pero por supuesto no son responsables de los errores aún presentes en este trabajo.

i Simonsen [1970] sostiene que en los últimos tres o cuatro años, la tasa de inflación podría correlacionarse únicamente con la tasa de cambio en la oferta monetaria y trata de resaltar el papel de los mecanismos de retroalimentación (como la indización) en el nivel de precios. Morley [1970] sugiere una explicación "híbrida", dando énfasis al papel dominante de la inflación por el lado de los costos en algunos períodos, y por el lado de la demanda en otros. 
Debido a causas que se aclararán luego, se supone que $m^{d}$ y $y$ están expresados en logaritmos naturales, y que $E_{t}$ es la tasa de inflación esperada.

La tasa de inflación esperada sigue al modelo de expectativas adaptativo sugerido por Cagan. ${ }^{2}$

$$
(1-b L) E_{t}=(1-b) \pi_{t-1}
$$

donde $L$ es el operador de retardos, y $\pi_{t}=\log P_{t}-\log P_{t-1}$ es la tasa de inflación real.

$\mathrm{Si}$ en cualquier momento deseado el saldo en efectivo real fuera igual a la demanda para un modelo monetario, éste se completaría con (2.1) y (2.2). Si existe la posibilidad de desequilibrio en la existencia del mercado monetario, y también la posibilidad de un tipo de flujo desequilibrado, en el sentido de que la tasa de cambio real de la existencia monetaria es diferente a la tasa deseada del saldo en efectivo real nominal, se necesita una ecuación de ajuste de la forma:

$m_{t}-m_{t-1}=(1-d)\left(m_{t}^{d}-m_{t-1}\right)+g\left[\mu_{t}=(1-L) \log M_{t}^{d}\right]$

donde $m_{t}$ es el logaritmo del saldo actual en efectivo

$\left(\log M_{t}-\log P_{t}\right)$.

$M_{t}^{d}$ es la existencia deseada nominal de fondos monetarios y $\mu_{t}=$ $\log M_{t}-\log M_{t-1}$ es la tasa de crecimiento de la oferta monetaria. La ecuación (2.3) tiene dos mecanismos de ajuste. El primer término del lado derecho es un tipo de ajuste nerloviano que muestra que si la existencia real es mayor (o menor) que la actual, la gente tratará de acumular (o de deshacerse) de saldos en efectivo reales en una proporción de $100(1-d) \%$ del incremento (o disminución) deseado. Se muestra que cualquier cambio en la demanda a largo plazo no provocará cambios inmediatos en los saldos en efectivo reales actuales, y reaccionarán con algunos retrasos. El segundo término del lado derecho muestra que si la tasa de crecimiento en la oferta monetaria nominal llega a ser mayor que la tasa a la cual la gente desea acumular saldos en efectivo nomínales, en el plazo muy corto, esta gente retendrá $100 \% g$ de este exceso en sus saldos reales en efectivo.

Podemos empezar desde la situación inicial de equilibrio pleno (de existencias y flujos) con $E$ y $Y$ consonantes. Si en el momento $t$ la tasa de crecimiento de la oferta monetaria $\mu_{t}$ llega a ser mayor que la tasa de crecimiento del saldo en efectivo nominal $(1-L) \log M^{d}$, y de $t+1$ en ambos vuelve a ser idéntica, al principio del proceso de ajuste, la propor-

${ }^{2}$ Muth [1960] mostró que bajo algunas hipótesis específicas acerca de las características estocásticas del modelo, esta ecuación produce el mejor predictor permanente de la inflación futura en el sentido de minimizar el error medio cuadrático de predicción. 
ción $g$ de la difetrencia $\left[\mu_{t}-(1-L) \log M^{d}\right]$ se considerará como un aumento del saldo actual real en efectivo (con $(1-g) \mu_{t}-(1-L)$ $\log M^{d}$ ) incrementando la demanda de bonos y bienes y servicios). Del siguiente período en adelante, se tendrá $m_{t-1}>m_{t}^{d}$ y el término nerloviano de (2.3) mostrará como puede ocurrir la convergencia hacia el nuevo equilibrio.

En el plazo muy corto, es posible que la población retendrá todo el exceso de $\mu$ sobre $(1-L) M^{d}$, y en el caso especial $g=1$, En un período más largo, debemos esperar $0 \leqslant g \leqslant 1$. Si el período de tiempo es lo suficientemente largo para permitir el ajuste pleno, tendremos que $g$ y $d$ convergerán a cero.

Un análisis más cuidadoso de este modelo, comparando su mérito relativo en relación con las especificaciones alternativas (como las propuestas por Chow, -en 1966 - por ejemplo) se efectua en otro trabajo. ${ }^{3}$

Para la manipulación más fácil del modelo la ecuación (2.3) puede simplificarse para analizar sus implicaciones en la dinámica de la inflación. La demanda de saldos en efectivo nominales puede expresarse en la forma

$$
\log M_{t}^{d}=\log P_{t}^{e}-\alpha E_{t}+\beta Y_{t}
$$

donde $P_{t}^{e}$ es el nivel de precios esperado (cuando la gente espera retener $\left.M_{d}\right)$. La tasa de cambio efectiva nominal deseada será.

$$
(1-L) \log M_{t}=(1-L) \log P_{t}^{e}-\alpha(1-L) E_{t}+\beta(1-L) Y_{t}
$$

Si la tasa de cambio en el ingreso real y la tasa de inflación esperada son pequeños y si se supone que la tasa de cambio del nivel de precios esperado es la tasa de inflación esperada, tendremos $(1-L) M_{t}^{d}-E_{t}$, y la ecuación (2.3) puede reescribirse de la siguiente forma:

$$
m_{t}-m_{t-1}=(1-d)\left(m_{t}^{d}-m_{t-1}\right)+g\left(\mu_{t}-E_{t}\right)
$$

la que está vinculada al modelo.

La implicación de esta hipótesis para la dinámica de inflación puede analizarse al aproximar el modelo a la ecuación cuantitativa que expresada en forma de tasa de crecimiento es:

$$
\pi_{t}=\mu_{t}(1-L) m_{t}
$$

De (2.1) y (2.3) obtenemos

$$
(1-d L) m_{t}=-\alpha(1-d) E_{t}+\beta(1-d) Y_{t}+g\left(\mu_{t}-E_{t}\right)
$$

y al usar (2.2) obtenemos

$$
\begin{aligned}
& (1-d L)(1-b L) m_{t}=-(1-b)[\alpha(1-d)+g] L \pi_{t} \\
& \quad+\beta(1-d)(1-b L) Y_{t}+\mathrm{g}(1-b L) \mu_{t}
\end{aligned}
$$

${ }^{3}$ Véase Pastore [1974]. 
El sistema (2.4) (2.6) puede expresarse en forma matricial:

$$
\begin{aligned}
& {\left[\begin{array}{cc}
(1-b L)(1-d L) & (1-b)[\alpha(1-d)+g] L \\
(1-L) & 1
\end{array}\right] \quad\left[\begin{array}{c}
m_{t} \\
\pi_{t}
\end{array}\right]}
\end{aligned}
$$

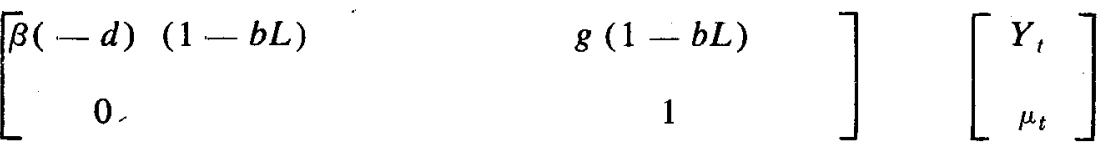

El modelo tiene una solución en la forma de "retardos racionales distribuidos", o sea:

$$
\begin{aligned}
& m_{t}=\frac{A_{1}^{1}(L)}{P(L)} Y_{t}+\frac{A_{2}^{1}(L)}{P(L)} \mu_{t} \\
& \pi_{t}=\frac{B_{1}^{1}(L)}{P(L)} Y_{t}+\frac{B_{2}^{1}(L)}{P(L)} \mu_{t}
\end{aligned}
$$

$P(L)$ es el determinante asociado a la matriz de coeficientes de las variables endógenas, que también corresponde a la ecuación característica de las dos ecuaciones diferenciales (2.7) y (2.8), lo que permite condiciones de estabilidad al análisis.

Para analizar el impacto inicial sobre $\pi$ del movimiento en $\mu$ o $y$, podemos usar los polinomios en (2.7) y (2.8) dados por:

$$
\begin{aligned}
& A_{1}^{1}(L)=\beta(1-d)-\beta b(1-d) L \\
& A_{2}^{1}(L)=g-\{g b+[\alpha(1-d)+g](1-b)\} L \\
& B_{1}^{1}(L)=\beta(1-d)+\beta(1-d)(1+b) L-\beta b(1-d) L^{2} \\
& B_{2}^{1}(L)=(1-g)-[b+d-g(1+b) L]+b(d-g) L^{2}
\end{aligned}
$$

En un equilibrio estacionario, cuando $y$ y $\mu$ han sido contantes por un período lo suficientemente largo para permitir un equilibrio pleno ( $\mathrm{si}$ éste es estable) tendremos:

$$
\frac{A_{1}^{1}(1)}{P(1)}=\beta ; \frac{A_{2}^{1}(1)}{P(1)}=-\alpha ; \frac{B_{1}^{1}(1)}{P(1)}=0 ; \frac{B_{2}^{1}(1)}{P(1)}=1
$$

lo que significa que la tasa de expansión monetaria de $1 \%$ producirá el $1 \%$ en la tasa de inflación. Un aumento del ingreso real del nivel constante a otro nivel mostrará sus efectos en la tasa de inflación sólo en el plazo intermedio.

Sin embargo los saldos en efectivo reales cambiarán $\beta$ veces el cambio en el nivel de ingreso. 
La estabilidad del equilibrio dependerá de dos soluciones para $P(L)$ dadas por

$$
\begin{gathered}
\frac{[b+d+(1-b) z] \pm \sqrt{[b+d+(1-b) z]^{2}-4[b d+(1-b) z]}}{2} \\
\text { en donde } z=\alpha(1-d)+g
\end{gathered}
$$

La tasa de inflación oscilará o no dependiendo de

$$
\{b+d+(1-b)[\alpha(1-d)+g]\}^{2} \gtrless 4\{b d+(1-b)[\alpha(1-d)+g]\}
$$

Toda vez que $b$ y $d$ sólo pueden tomar valores entre cero y uno la estabilidad del modelo es dada por: ${ }^{4}$

$$
b d+(1-b)[\alpha(1-d)+g]<1
$$

\section{EVIDENCIAS EMPÍRICAS}

La demanda monetaria expresada en (2.5) se estimó suponiendo valores diferentes para el coeficiente de expectativas $(1-b)$ en la forma:

$$
m_{t}=-[\alpha(1-d)+g] E_{t}+\beta(1-d) Y_{t}+g_{\mu_{t}}+d m_{t-1}+w_{t}
$$

\begin{tabular}{|c|c|c|c|c|c|c|c|}
\hline \multirow{2}{*}{$\begin{array}{l}\text { Palor do } \\
(1-b)\end{array}$} & \multicolumn{5}{|c|}{ Coopioiente } & \multirow{2}{*}{$\mathrm{B}^{2}$} & \multirow{2}{*}{$D W^{b} /$} \\
\hline & oonatanto & $\mathbf{s}_{t}$ & $r_{t}$ & $m_{t-1}$ & $H_{t}$ & & \\
\hline 0.2 & 0.759 & $\begin{array}{l}-0.691 \\
(3.756)\end{array}$ & $\begin{array}{c}0.130 \\
(2.077)\end{array}$ & $\begin{array}{c}0.847 \\
(10.027)\end{array}$ & $\begin{array}{c}0.605 \\
(3.874)\end{array}$ & 0.950 & 1.354 \\
\hline 0.3 & 1.202 & $\frac{-0.917}{(5.075)}$ & $\begin{array}{c}0.178 \\
(2.986)\end{array}$ & $\begin{array}{c}0.773 \\
(9.494)\end{array}$ & $\begin{array}{c}0.666 \\
(4.585)\end{array}$ & 0.958 & 1.530 \\
\hline 0.4 & 1.505 & $\frac{-1.059}{(5.917)}$ & $\begin{array}{c}0.185 \\
(3.342)\end{array}$ & $\begin{array}{c}0.736 \\
(9.391)\end{array}$ & $\begin{array}{c}0.676 \\
(4.953)\end{array}$ & 0.962 & 1.814 \\
\hline 0.5 & 1.464 & $\begin{array}{l}-0.945 \\
(5.904)\end{array}$ & $\begin{array}{c}0.177 \\
(3.233)\end{array}$ & $\begin{array}{c}0.745 \\
(9.580)\end{array}$ & $\begin{array}{c}0.640 \\
(4.736)\end{array}$ & 0.962 & 1.932 \\
\hline 0.6 & 1.384 & $\begin{array}{l}-0.854 \\
(5.810)\end{array}$ & $\begin{array}{c}0.165 \\
(3.041)\end{array}$ & $\begin{array}{c}0.760 \\
(9.850)\end{array}$ & $\begin{array}{c}0.607 \\
(4.504)\end{array}$ & 0.962 & 2.031 \\
\hline 0.7 & 1.277 & $\begin{array}{l}-0.777 \\
(5.607)\end{array}$ & $\begin{array}{c}0.150 \\
(2.772)\end{array}$ & $\begin{array}{c}0.779 \\
(10.128)\end{array}$ & $\begin{array}{c}0.571 \\
(4.211)\end{array}$ & 0.960 & 2.118 \\
\hline 0.8 & 1.160 & $\begin{array}{r}-0.706 \\
5.472\end{array}$ & $\begin{array}{c}0.135 \\
(2.525)\end{array}$ & $\begin{array}{c}0.800 \\
(10.488)\end{array}$ & $\begin{array}{c}0.561 \\
(4.101)\end{array}$ & 0.959 & 2.180 \\
\hline 0.9 & 1.040 & $\begin{array}{l}-0.645 \\
(5.300)\end{array}$ & $\begin{array}{c}0.120 \\
(2.261)\end{array}$ & $\begin{array}{c}0.821 \\
(10.822)\end{array}$ & $\begin{array}{c}0.553 \\
(4.000)\end{array}$ & 0.959 & 2.233 \\
\hline
\end{tabular}

donde $w_{t}$ es el término perturbador suponiendo que no está correlacionado en serie.

Cuadro 1

DEMANDA MONETARIA

" Los números entre paréntesis corresponden al valor de la $t$ de student.

b Prueba Durwin Watson.

‘ Véase a Goldberg [1961, pp. 169 a 172]. 
La dificultad para usar el método iterativo en este caso se debe a la presencia de retardos en los saldos en efectivo reales como una variable independiente en el modelo, lo que convierte a $R^{2}$ insensible a las variaciones en $(1-b) .5$

Los resultados se presentan en el cuadro 1. Como puede observarse, todos los coeficientes son muy significativos, y $R^{2}$ alcanza su máximo aproximadamente hasta el punto donde $(1-b)=0.4$, que se considera como un estimador del coeficiente de expectativas.

No existen razones para rechazar la hipótesis de que el equilibrio es estable, y de que la trayectoria de convergencia es oscilatoria.

El cuadro 2 y la gráfica 1 , muestran la trayectoria típica de la tasa de inflación en una situación hipotética, donde la economía se aparta de una situación previa de equilibrio, con tasas de inflación de $0 \%$ trimestral, hasta una nueva situación donde la tasa de expansión monetaria es constante al $1 \%$ trimestral. La parte (A) de la gráfica muestra la trayectoria de la tasa de inflación.

La tasa de inflación tomará alrededor de tres a cuatro trimestres para sobrepasar la tasa de expansión monetaria, lo que significa que los efectos iniciales de la oferta monetaria sobre los precios son mucho menores y que los retrasos son mayores.

El modelo admite una "ecuación reducida" (mediante la expansión de polinomios de $L$ en (2.8)) que expresan la tasa de inflación real como una función de las tasas anteriores de crecimiento de la oferta monetaria $y$ del ingreso real en la forma de:

$\pi_{t}=\sum_{j=o}^{\infty} w_{j}^{1} \mu_{t-j}-\beta \sum_{j=o}^{\infty} w_{j}^{2}(1-L) y_{t-j}$

donde $w_{j}^{i}$ es la ponderación del modelo implícito con retardos distribuidos en $\pi$ (variable dependiente), y los valores pasados de $\mu$ y $(1-L) y$ (variable independiente). El esquema de las ponderaciones se presenta en el cuadro 2 y parte $B$ de la gráfica en que se muestra que después de algún retardo de tiempo se obtienen ponderaciones negativas. Tal resultado es consistente con la existencia de demanda monetaria dependiendo ésta del costo de la retención de fondos.

5 La ecuación que se estima es:

$$
m_{t}=-[\alpha(1-d)+g] E_{t}+\beta(1-d) y_{t}+d m_{t-1}+g \mu_{t}
$$

De (2.2) puede expresarse $E_{t}$ como una media móvil de los valores pasados de $\pi$ con ponderaciones geométricamente decrecientes; $b$ puede estimarse por el método iterativo de máxima verosimilitud (véase a Cagan -1956-) y en particular el apéndice). De (2.2) se tiene $E_{t}=(1-b) \pi_{t-1}+b E_{t-1}$ y al substituir este valor en la expresión anterior se obtiene $m_{t}=-[a(1-d)+g](1-b) \pi_{t-1}-a(1-d) b E_{t-1}$ $\left.+\beta(1-d) y_{t}+d m_{t-1}+g \mu^{t}+W_{t}\right)$. En el caso extremo de $b=0$, las tasas de inflación reales y esperadas serán iguales y $E_{t-1}$ desaparecería de esta expresión. Si $b$ es diferente de cero, se excluye $E_{t-1}$ por error de la ecuación, debido a la correlación entre $E_{t-1}$ y $m_{t-1}$ esta última variable absorberá parte de la explicación debido a $\mathrm{E}_{t^{-1}}$ sesgando la estimación de $L S$ en $m_{t-1}$. Esto muestra que cuando $m_{t-1}$ está incluído explícitamente en el modelo, $R^{2}$ será insensible a la interacción de $b$. 
Imaginemos una situación inicial donde la taśa de inflación estaba en equilibrio (con un saldo en efectivo real constante) con $\mu=\pi$ al nivel de $0 \%$ por trimestre. Si las autoridades monetarias decidieran efectuar un cambio de una vez por todas en $\mu$ de $1 \%$ por trimestre y se fijara a este nuevo nivel, la población sería relativamente ineficiente, en el corto plazo, para deshacerse del exceso de oferta monetaria.

Después de algún tiempo, la tasa de inflación esperada comienza a crecer, se incrementá el costo de retención de fondos, se reduce la existencia monetaria real y se agregan nuevas presiones inflacionarias. Al final del proceso, la tasa de inflación y el costo de retención de fondos, por necesidad será más elevado, dado el actual acervo monetario (igual al deseado) pero más baja que en las situaciones de equilibrios anteriores. Así que después de algún tiempo, posterior al cambio en $\mu$, la tasa de inflación tiene que exceder $\mu$, manteniendo elevada esta tasa el tiempo necesario para reducir la existencia del saldo en efectivo real, hasta llegar a ser igual a la existencia deseada. Este mecanismo de ajuste implica que la relación entre la tasa de inflación en $t$ y las tasas anteriores configuran un esquema de distribución de retardos en el tiempo con ponderaciones negativas, que ya han sido establecidos por Diz [1970] para Argentina y por Pastore [1973a] para Brasil.

Cuadro 2

PONDERACIONES DEL MODELO IMPLíctTo DE RETARDos DISTRIBUÍDOS Y TRAYECTORIA DE $\pi t$

\begin{tabular}{|c|c|c|}
\hline \multirow{2}{*}{$\underset{j}{\text { Rotardos }}$} & \multicolumn{2}{|c|}{$\pi t$} \\
\hline & ponderaoionen us & trayootoria den \\
\hline $\begin{array}{c}0 \\
1 \\
2 \\
3 \\
4 \\
5 \\
6 \\
7 \\
8 \\
9 \\
10 \\
11 \\
12 \\
13 \\
14 \\
15 \\
16 \\
17 \\
18 \\
19 \\
20 \\
21 \\
22 \\
23 \\
24 \\
25\end{array}$ & $\begin{array}{r}0.324 \\
0.316 \\
0.311 \\
0.273 \\
0.211 \\
0.135 \\
0.055 \\
-0.020 \\
-0.083 \\
-0.089 \\
-0.129 \\
-0.155 \\
-0.161 \\
-0.149 \\
-0.142 \\
-0.083 \\
-0.087 \\
-0.046 \\
-0.006 \\
0.029 \\
0.056 \\
0.074 \\
0.082 \\
0.080 \\
0.070 \\
0.054 \\
0.034 \\
0.013\end{array}$ & $\begin{array}{l}0.324 \\
0.640 \\
0.951 \\
1.224 \\
1.435 \\
1.570 \\
1.625 \\
1.605 \\
1.522 \\
1.393 \\
1.238 \\
1.077 \\
0.928 \\
0.805 \\
0.718 \\
0.0672 \\
0.666 \\
0.6955 \\
0.751 \\
0.825 \\
0.907 \\
0.987 \\
1.057 \\
1.111 \\
1.145 \\
1.158\end{array}$ \\
\hline
\end{tabular}

- Las ponderaciones y la trayectoria de $\pi$ se calcularon con los siguientes valores de los coeficientes:

$\beta=0.701 ; g=0.676 ; d=0.736 ; b=0.600 ; \alpha=(1-\mathrm{d})+g=1.059$ 
PASTORE: POLITICA MONETARIA DE BRASIL

331

Gráfica 1

Trayectoria DE $\pi$ Y ESQUEMa DE Distribución DE RETARdos EN EL TIEMPO ENTRE $\pi$ Y LOS VALORES PASADOS DE $\mu$
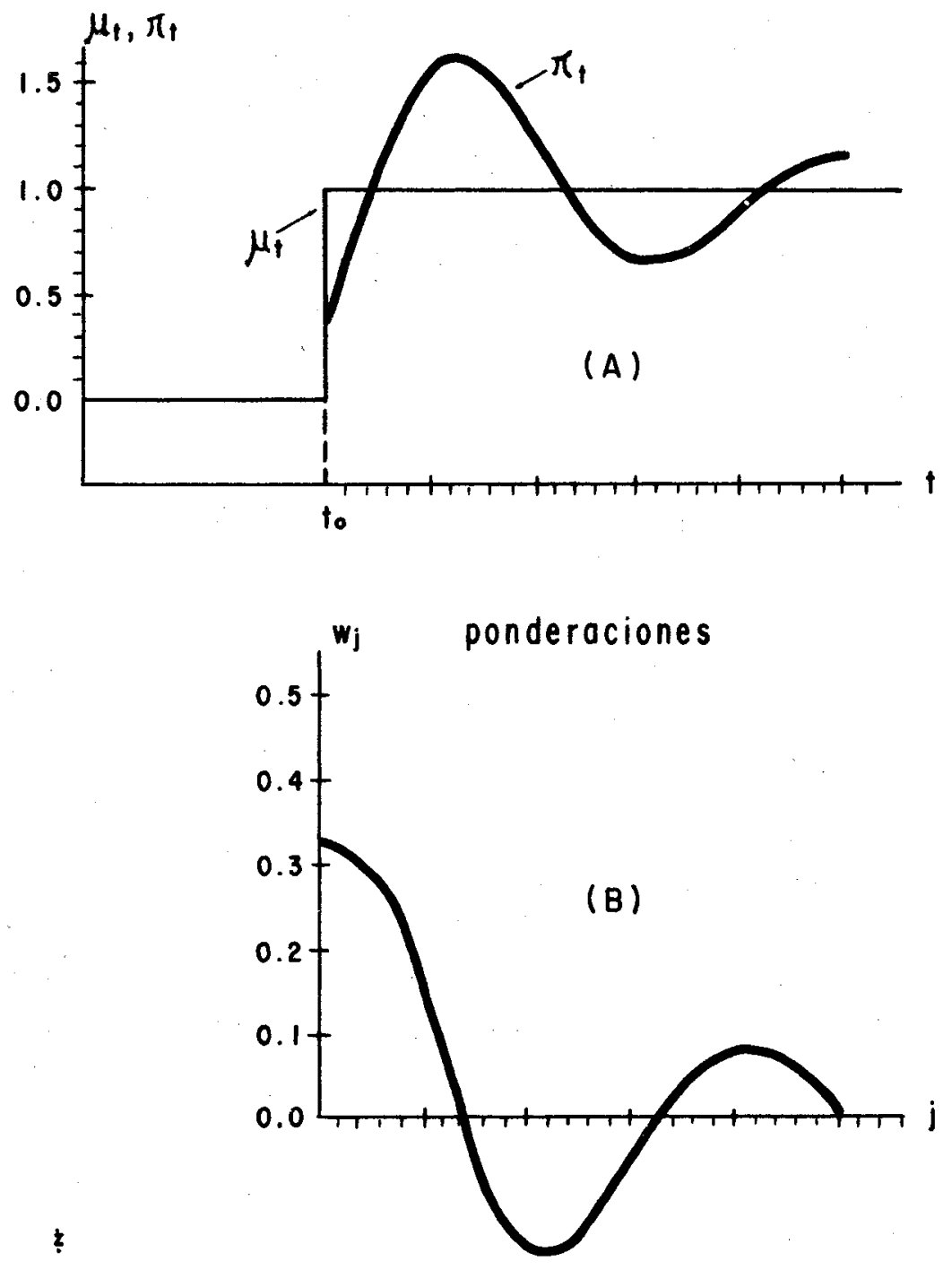


\section{LA INFLACIÓN BRASILEÑa}

¿Sería posible explicar la inflación brasileña incluyendo sólo las variables monetarias? ¿hasta dónde se puede atribuir a otras "variables no monetarias" alguna capacidad explicativa en el comportamiento del nivel general de precios?

Existe un cierto consenso de que la tasa de inflación en Brasil está afectada por lo menos por tres variables adicionales: a) los reajustes en la tasa de salarios (salarios medios y salarios mínimos en particular); b) corrección del tipo de cambio; c) fluctuaciones de la oferta agrícola. Se podría añadir una cuarta variable que con seguridad fue importante durante la primera parte del programa de estabilización brasileño, que consiste en liberar algunos precios que habían sido congelados como práctica para controlar la inflación. Algunas de estas variables pueden tener efectos cuantitativos mientras que otras no los tienen. En particular, sólo existe información disponible para salarios mínimos y tipos de cambio, pero no existe información trimestral sobre la oferta agrícola y sobre la forma de procesamiento de la llamada "inflación correctiva". Si se usa $\hat{\pi}$, para la tasa estimada de inflación sólo como una función de componentes monetarios, o sea $\widehat{\pi}_{t}=\mu_{t}-(1-L) \widehat{m}_{t}$, donde $\widehat{m}_{t}$ es el saldo en efectivo real estimado en la ecuación (2.5), podemos verificar la influencia de las demás variables con la relación:

$$
\pi_{t}=f\left(\hat{\pi}_{t}, \dot{c}_{t}, \dot{w}_{t}\right)+v_{t}
$$

donde $\dot{w}_{t}$ y $\dot{c}_{t}$ representan la tasa de cambio en los salarios mínimos y el tipo de cambio.

El cuadro 3 muestra los principales resultados. La primera relación es la simple regresión entre la tasa de inflación trimestral real y estimada. La segunda expresa la misma relación pero definida como la tasa de inflación real anual al final del trimestre $t$.

Cuadro 3

TASAS DE INFLACIÓN OBSERVADAS Y ESTIMADAS ${ }^{\text {a }}$

\begin{tabular}{|c|c|c|c|c|c|c|c|c|}
\hline \multicolumn{4}{|c|}{ Coeficienter } & \multirow{2}{*}{$\mathrm{R}^{2}$} & \multirow{2}{*}{ DH } & \multirow{2}{*}{$\begin{array}{l}\text { Variable } \\
\text { depen- } \\
\text { riiente }\end{array}$} & \multirow{2}{*}{$\begin{array}{l}\text { Defini- } \\
\text { cion } \\
\text { de tasa }\end{array}$} & \multirow{2}{*}{$\begin{array}{l}\text { Ecuación de } \\
m_{t}\end{array}$} \\
\hline Constentes & $\pi$ & $\dot{i}$ & $\dot{0}$ & & & & & \\
\hline 0.020 & $\begin{array}{c}0.668 \\
(5.782)\end{array}$ & - & - & 0.406 & 2.407 & $\pi$ & b/ & $(19) \operatorname{con}(4-b)=0.4$ \\
\hline 0.015 & $\begin{array}{r}0.966 \\
(21.084)\end{array}$ & - & - & 0.901 & 1.180 & $\pi$ & of & (19) oon $(1-b)=0.4$ \\
\hline 0.031 & $\begin{array}{c}0.607 \\
(5.782)\end{array}$ & - & - & 0.405 & & $\hat{\pi}$ & $\underline{b}$ & $(19) \operatorname{con}(1-b)=0.4$ \\
\hline 0.031 & $\begin{array}{c}0.479 \\
(5.091)\end{array}$ & $\begin{array}{c}0.151 \\
(4.417)\end{array}$ & - & 0.577 & & $\hat{\pi}$ & b/ & $(19) \operatorname{con}(1-b)=0.5$ \\
\hline 0.022 & $\begin{array}{c}0.494 \\
(5.694)\end{array}$ & $\begin{array}{c}0.126 \\
(3.893)\end{array}$ & $\begin{array}{c}0.120 \\
(3.080)\end{array}$ & 0.648 & 2.070 & $\hat{\pi}$ & b & $(19) \operatorname{con}(1-b)=0.5$ \\
\hline
\end{tabular}

a Los números entre paréntesis corresponden a los valores del estadístico $t$

b Trimestral

c Anual y fin de trimestre 
El coeficiente de regresión entre $\pi$ y $\hat{\pi}$ es menor que uno, y sólo al reducir la "significancia" por abajo del $5 \%$, el intervalo de confianza incluiría a la unidad (sólo en el caso de tasas trimestrales).

Las dos últimas ecuaciones muestran la introducción de la tasa de salarios y el tipo de cambio. Ambas tienen coeficientes de alta significación que muestran que no existen razones para rechazar la hipótesis de que al menos dos de las variables "remanentes", influyen en la tasa de inflación. El poder explicativo del modelo es más alto cuando se incluyen tasas de salarios y tipos de cambio. ${ }^{6}$

Debido a los efectos de retroalimentación (a través de las expectativas), los efectos de los salarios o corrección de los cambios sobre la tasa de inflación, se esparcirán a lo largo de muchos períodos. De hecho, se puede reescribir (2.4) en la forma:

$$
\pi_{t}=\mu_{t}-(1-L) m_{t}+\sigma_{t}
$$

donde

$$
\sigma_{t}=a_{1} \dot{w}_{t}+a_{2} \dot{c}_{2}+e_{t}
$$

es una combinación lineal de efectos de las "otras variables" que afectan $\pi$ y los coeficientes $a_{1}$ y $a_{2}$ son los estimados en el cuadro 3. El sistema (4.2), (4.3) puede expresarse en forma matricial:

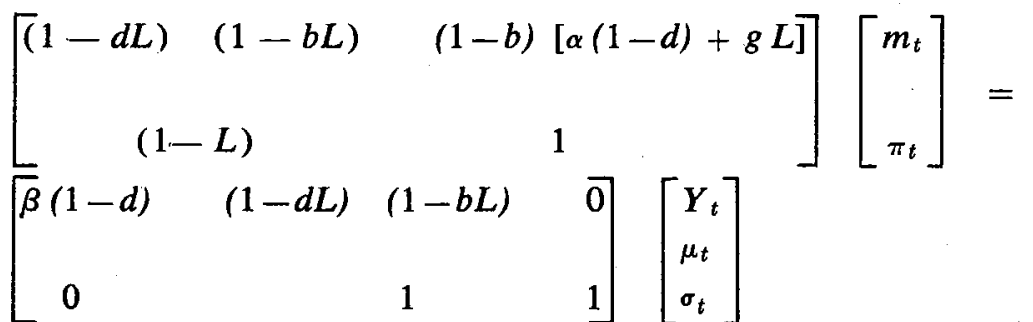

que permiten encontrar la expresión para la tasa de inflación dada por:

$$
P(L) \pi_{t}=B_{1}^{1}(L) Y_{t}+B_{2}^{1}(L) \mu_{t}+B_{3}^{1}(L) \sigma_{t}
$$

donde todos los polinomios ya han sido definidos excepto $B_{3}^{1}(L)$, o sea:

$$
B_{3}^{1}(L)=1-(b+d) L+b d L
$$

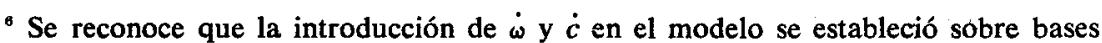
muy ad-hoc, sin la especificación del modelo subyacente. La teoría podría desarrollarse de varias formas, pero creo que la más atractiva es la sugerida por Harberger [1966] en la que se establece que en un modelo general de equilibrio, donde existen variables reales (ingresos) y variables nominales (el nivel de precios general) como variables endógenas y variables nominales (oferta monetaria, salarios y tipos de cambio) como exógenas, la ecuación del modelo en su "forma reducida será homogénea de grado uno, lo que expresa las endógenas nominales como función de las exógenas y homogéneas de grado cero, expresando las endógenas reales como una función de las exógenas. En este caso, la relación (4.1) podría interpretarse como una ecuación reducida del modelo, verificando que al aumentar las elasticidades de $\pi$ respecto a las variables nominales se obtiene un número muy cercano a uno 
Gráfica 2

IMPACTO DE LA TASA DE SALARIOS O DE LA CORRECCIÓN DEL TIPO DE CAMBIO SOBRE LA TASA DE INFLACIÓN

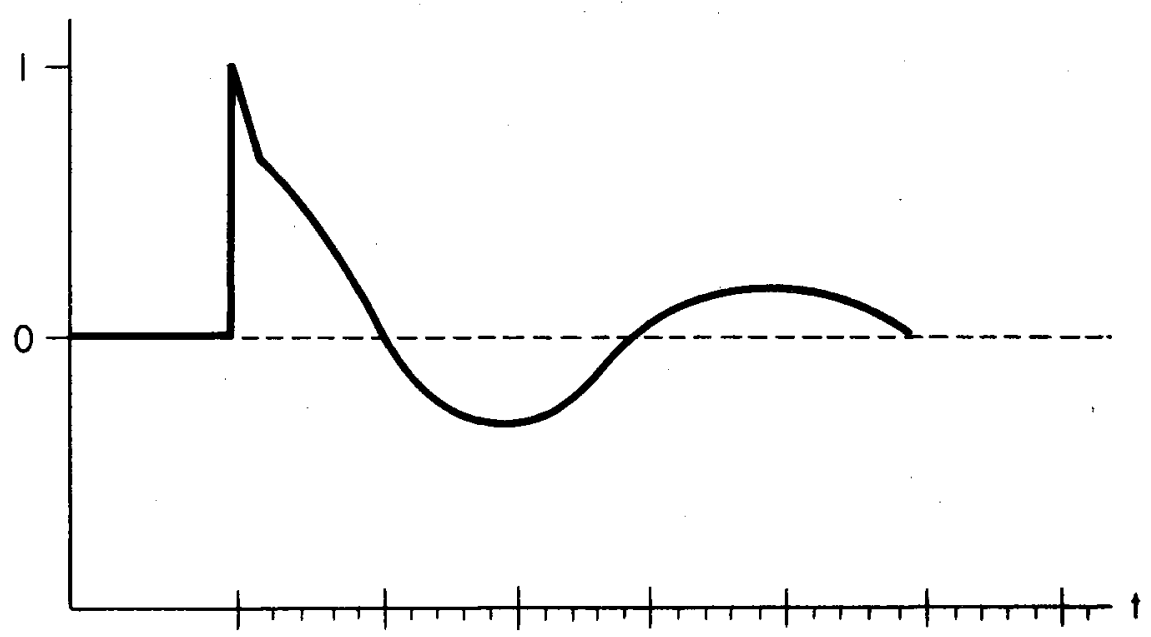

Es fácil advertir que $P(1) / B_{3}^{1}(1)=1$, que indica que el efecto final de l̀os salarios y los reajustes en los tipos de cambio sobre $\pi$ estarán dados, en cada caso, por el coeficiente respectivo de la relación (4.1). Sin embargo, un cambio en las tasas de salarios o tipos de cambio, causarán efectos retardados en la tasa de inflación, cuya trayectoria en el tiempo puede calcularse con facilidad, ceteris paribus, mediante:

$$
P(L) \pi_{t}=B_{3}^{1}(L) \sigma_{t}
$$

La gráfica 2 muestra el esquema típico de esta trayectoria calculado con los coeficientes estimados en (2.5).

Adviértase que no sólo se tiene un impacto inicial sino que debido a la retroalimentación (generada por las expectativas), la "presión de los costos" permanece positiva durante muchos períodos. Por lo tanto, oscila alrededor de cero (al suponer que cero era el equilibrio inicial de la tasa de inflación y que las otras variables permanecieron constantes).

La sucesión de choques puede producir graves efectos, no sólo cuando ocurre cada uno de ellos, sino también durante muchos períodos. Si la "inflación controlada" es sustancial, la economía encarará un proceso inflacionario "autónomo" que no sólo ocurrirá en el momento de la corrección, sino generará efectos sobre $\pi$ por un período más largo. 
Gráfica 3

VALORES REALES Y ESTIMADOS DE $\pi$ Y $m$

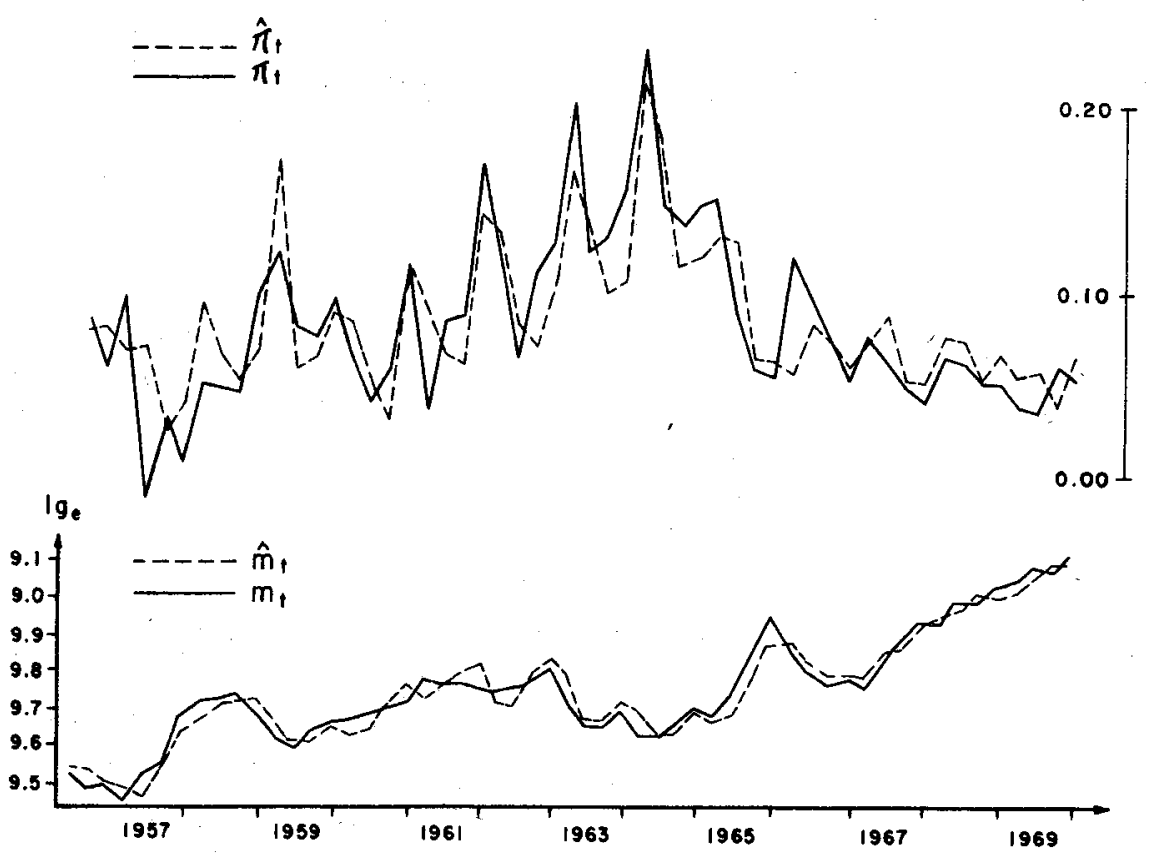

La gráfica 3 muestra la comparación entre los valores actuales y los de la predicción de $\pi$ y $m$.

\section{ASPECtos DE LA ESTRATEGIA DE ESTABILIZACIÓN}

Una implicación importante del análisis anterior es que desde el punto de vista de la tasa de inflación, el dinero sólo importa en el largo plazo. Aún así, no se trata de cuestionar la precisión de los retardos en el tiempo 7 estimados. Existen evidencias de que tales retardos en el tiempo son bastante largos, y esto probablemente es la principal razón de la presencia de ciclos muy largos en la velocidad del ingreso y en el saldo en efectivo real. En el muy corto plazo, un aumento en la tasa real de expansión monetaria, incrementará el saldo en efectivo real sobre el nivel de equilibrio previo, y la velocidad del ingreso monetario estimado sufrirá

- El análisis de Griliches [1967] muestra que los retardos son altamente sensibles a los valores estimados de los coeficientes del modelo. 
una caída, simulando un comportamiento similar al de la "trampa de liquidez".

Cuando $\mu$ empieza a ascender, es muy probable que la tasa de inflación se quede por algún tiempo debajo de $\mu$. Entonces es cuando se empiezan a revisar las expectativas, y la población logrará deshacerse del saldo en efectivo real. Si las autoridades monetarias establecieran eventualmente $\mu$ a nivel constante durante algún período futuro $\pi$ sobreexcederá a $\mu$.

Esto demuestra que la correlación simultánea mensual y aún trimestral entre $\pi$ y $\mu$ probablemente será muy reducida, y las autoridades monetarias podrían sufrir el espejismo de que el dinero no importa del todo, acusando a la inflación de ser un fenómeno puramente de presión de los costos.

Debido a la tendencia general de los gobiernos a combatir la inflación a través de sus manifestaciones y no a través de sus causas principales, cuando empieza la inflación abierta, también empieza la inflación controlada que se hace a través del control de ciertos precios (en general los "precios políticos", por ejemplo, los artículos y servicios que tienen más peso en algún índice que se considera como una medida de la inflación). Tal procedimiento introduce toda clase de distorsiones en los precios relativos, lo que causa ineficiencias y muestra que después de algún tiempo esta inflación controlada tendrá que ser absorbida por la economía. Cuando el gobierno decide enfrentar el problema de estabilización, deberá absorber la inflación controlada liberando los precios sujetos a control, dando lugar a la llamada "inflación correctiva". En la gráfica 4 pueden analizarse las posibles situaciones de este caso.

La gráfica 4-A representa las estrategias de "aproximación sucesiva", presión monetaria y "tratamiento de choque" si no hay inflación controlada. Empecemos con la posición de un equilibrio inicial donde la expansión monetaria se fijó en $\mu_{0}$ y con la tasa de inflación al mismo nivel (suponiendo un ingreso real constante). La trayectoria de $(M / P)$ y $\pi$ se presenta en la gráfica entre los puntos $a$ y $b$ con la tasa de inflación y el saldo en efectivo real que sigue una trayectoria cíclica. Si las autoridades monetarias pueden sostener $\mu$ en el nuevo nivel $\mu_{1}$ por un período lo suficientemente largo, descenderá la tasa de inflación, pero con grandes fluctuaciones cíclicas en los saldos en efectivo reales. Las trayectorias graduales implican una pequeña reducción que permanecerá hasta que la tasa de inflación converga alrededor del punto de equilibrio y sólo entonces se hará una nueva reducción en $\mu$. La reducción de la tasa de inflación será muy lenta pero la oscilación en $(M / P)$ será aún menor.

La gráfica 4-B muestra al principio el tratamiento de choque en una economía que ha absorbido la inflación controlada. Si las autoridades monetarias liberan los precios controlados, se incrementaría la tasa de inflación reduciendo los saldos en efectivo reales. En este caso, pueden ocurrir dos alternativas. En primer lugar, $\mu$ se mantiene al mismo nivel que $\pi_{o}$ permaneciendo hasta que la tasa de inflación converge alre- 
Gráfica 4

TRAYECTORIA DE M/P Y $\pi$ BaJo DIVERSAS hiPótesis SOBRE LA CONDUCTa

DE $\mu$ Y DE LA INFLACIÓN CORRECTIVA

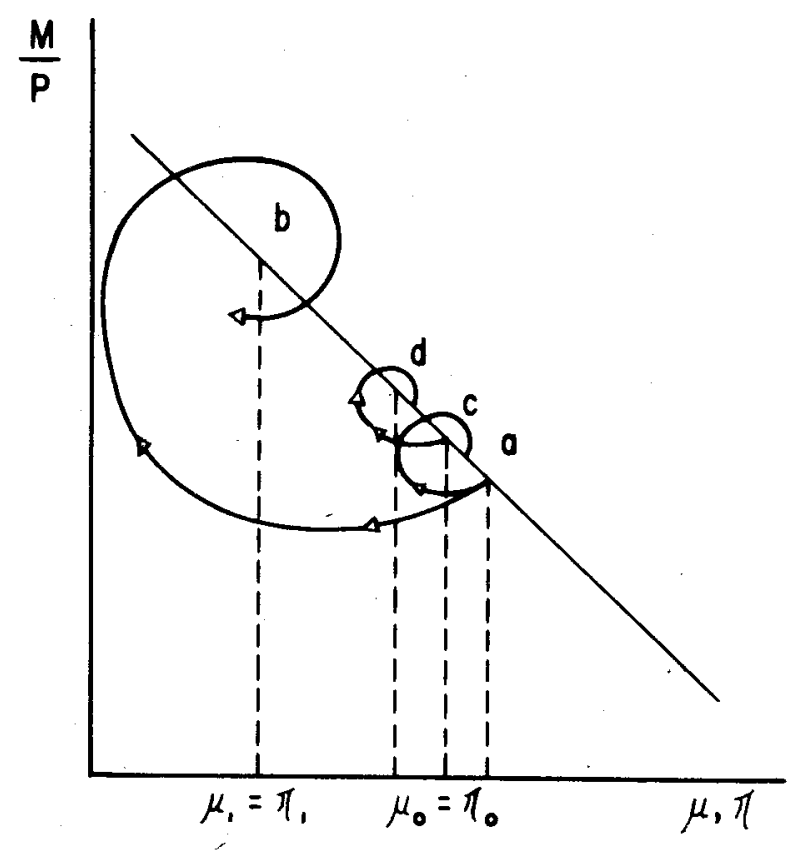

(A)

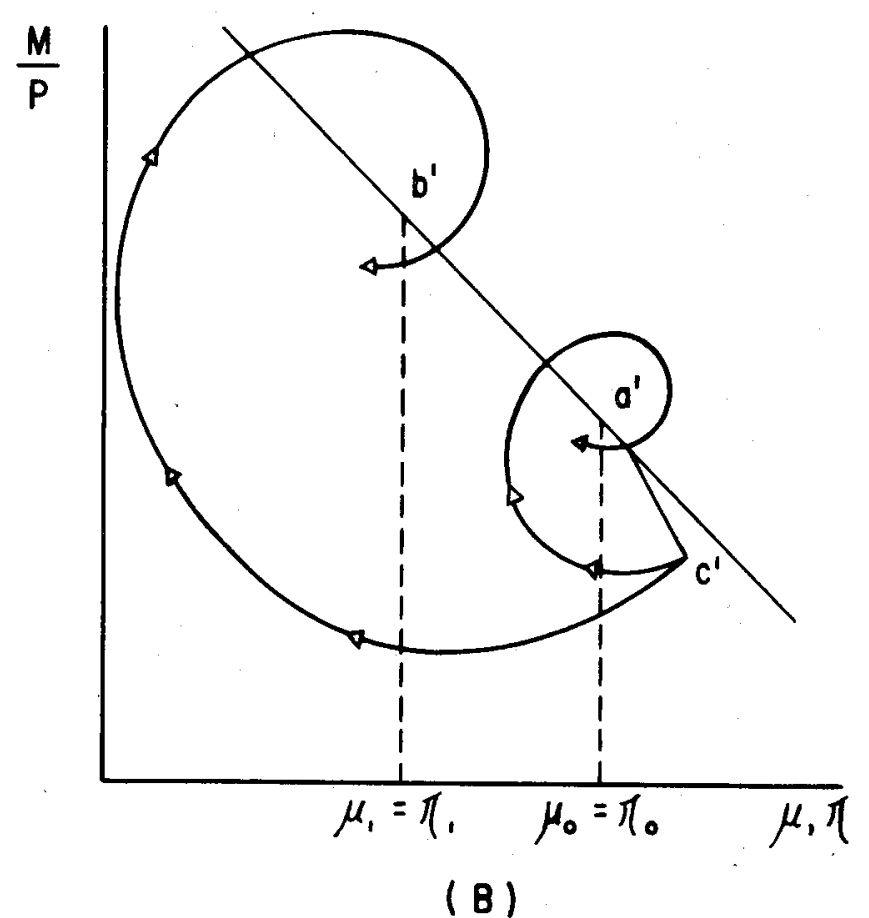


dedor del punto de equilibrio $a^{\prime}$. En este caso, pueden ocurrir algunos cíclos de liquidez. En la segunda alternativa, las autoridades monetarias procederían a una inflación correctiva, pero tratarían de evitar un incremento mayor de la tasa de inflación a través de la reducción en $\mu$. En este caso, $\pi$ y $(M / P)$ oscilarán alrededor de $b^{\prime}$.

La gráfica muestra la trayectoria seguida por $\pi$ y $(M / P)$, y resulta fácil observar que los ciclos en las dos variables son de una amplitud mucho mayor.

Es comprensible que no se efectúe la inflación correctiva simultáneamente con el choque monetario, y que el gobierno tiene que absorber primero la inflación controlada y a partir de entonces empezar la reducción gradual de la tasa de inflación.

\section{OfERTA MONetaria}

Al contrario de la práctica común, en otros países donde la emisión de fondos a través de la expansión de la demanda y los depósitos a plazo pueden hacerse a través de bancos comerciales, esta función en Brasil se ejerce simultáneamente por los bancos comerciales y las autoridades monetarias. Esto se debe al hecho de que en Brasil las autoridades monetarias están compuestas por dos instituciones bancarias: el Banco de Brasil que es al mismo tiempo un banco comercial y un agente financiero de las autoridades monetarias, y el Banco Central.

Por lo tanto, los pasivos monetarios netos de las ąutoridades mone tarias en poder del público, se obtienen al sumar los depósitos a plazo y la demanda de depósitos del público en el Banco de Brasil más la situación de reserva neta de los bancos comerciales, más el efectivo en poder del público:

$$
B=D^{B B}+R+M_{P}
$$

$\mathrm{Si} B$ designa la base monetaria y $k$ el multiplicador monetario, puede expresarse:

$$
M=k B
$$

La expresión para el multiplicador monetario puede obtenerse al definir el dinero como:

y luego

$$
M=M_{P}+D
$$

$$
k=\frac{1}{1-(1-h)(1-r)(1-b)}=\frac{1}{1-\delta}
$$

donde $M_{P}$ es la moneda circulante; $D$ corresponde al total de depósitos en los bancos comerciales y el Banco de Brasil, $D=D^{B B}+D^{B C} ; r$ es la tasa de reserva (voluntaria más obligatoria), $R / D^{B C}=r$; donde $r=r_{v}+r_{c} ; h$ es la tasa de circulante del total de la oferta monetaria, $M_{P} / M=h ; b$ es la propensión media del público a hacer depósitos en el Banco de Brasil, $h=P^{B B} / D$. 
Los dos instrumentos de política monetaria, en este modelo, son la base monetaria global y los requerimientos de la reserva oblagatoria $r_{c}$; los otros coeficientes $h, r$ y $b$ dependen del comportamiento de la población en el sistema bancario.

Dados los valores de los instrumentos bajo control de las autoridades monetarias y al predecir los valores de los coeficientes que dependerán del comportamiento de la población y del sistema bancario, puede obtenerse una predicción razonable del comportamiento de la oferta monetaria nominal.

Si se empieza de la relación (6.2) puede escribirse:

$$
\frac{1}{M} \frac{d M}{d t}=\frac{1}{m} \frac{d m}{d t}+\frac{1}{B} \frac{\mathrm{d} B}{d t}
$$

que divide el tipo de cambio de la oferta monetaria en la suma del tipo de cambio del multiplicador monetario y el tipo de cambio en la base monetaria. La gráfica 5 muestra las tasas de crecimiento de la base monetaria, el total de la oferta monetaria y el multiplicador monetario.

Puede observarse que la tasa de cambio del multiplicador monetario no es del todo pequeña, pero no hay duda que el componente dominante es la tasa de cambio de la base manetaria. ${ }^{8}$

¿Cuál es entonces la fuente de crecimiento de la base monetaria? ¿cuál es el mecanismo de control utilizado durante las distintas fases del programa de estabilización brasileña?

Para este propósito deben analizarse los activos del balance consolidado de las autoridades monetarias. Empecemos por: $B=\sum_{j=1}^{n} B_{j}$ donde $B_{j}$ es uno de los posibles activos de las autoridades monetarias. Es posible desagregar el tipo de cambio de la base monetaria (por el lado de los activos de las autoridades monetarias) en:

$$
\frac{1}{B} \frac{d B}{d t}=\sum_{j=1}^{n} \delta_{j}\left(\frac{1}{B_{j}} \cdot \frac{d B_{j}}{d t}\right)
$$

donde $\delta=B_{j} / B$ es la participación de cada activo en la base total y cada término $\delta_{j}\left(1 / B_{j}\right)\left(d B_{j} / d t\right)$ es la contribución del tipo de cambio de cada partida de activo en la base monetaria.

Podrían agregarse los distintos activos en la forma convencional: crédito total al gobierno; crédito total al sector privado y la posición del tipo de cambio neto, pero en virtud de ciertas peculiaridades del sistema institucional brasileño, se desagregarán los activos. Se proporciona ahora una breve explicación del significado de cada uno de ellos:

a) Déficit de tesorería: es la posición del efectivo neto acumulado del gobierno federal;

${ }^{8}$ Algunos de los coeficientes del multiplicador monetario $(h$ y $r$ ) son "parámetros de comportamiento", dependientes de las decisiones de maximización de ganancias del sistema bancario y del público. No se trató de incluir un submodelo para explicar $r$ y debido a que las monedas fuertes son el componente dominante de $M$. 


\section{Gráfica 5}

DESCOMPOSICIÓN DE LA TASA TRIMESTRAL

DE EXPANSIÓN MONETARIA

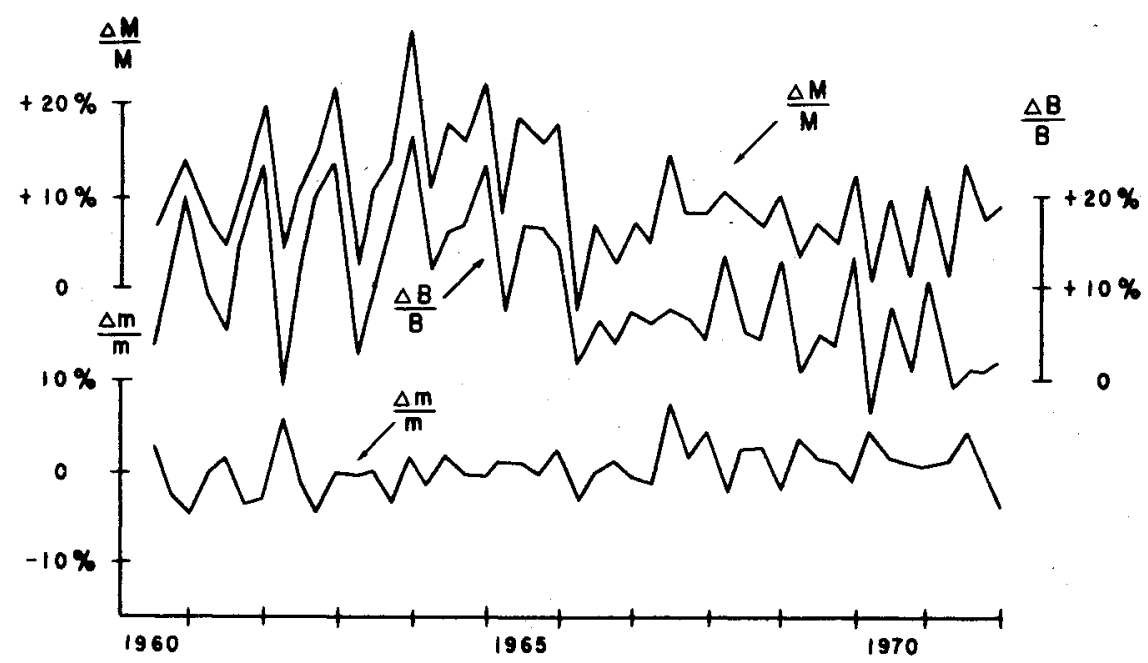

b) Bonos indizados: es el saldo neto del conjunto de operaciones cuya naturaleza ha estado cambiando a través del tiempo. Antes de la corección monetaria no había reajuste de bonos, y no era posible venderlos a largo plazo debido al alto nivel de inflación y a la congelación de la tasa de interés. Sin embargo, se emitieron los llamados "bonos de tesorería" que se colocaron primero en los bancos comerciales como substitutos de los depósitos obligatorios. En realidad esta partida incluye la venta de bonos indizados más los bonos de la tesorería (este es un mecanismo de endeudamiento a corto plazo para operaciones de mercado abierto);

c) Préstamos al sector privado por parte del Banco de Brasil: en esta partida se incluyen todos los préstamos a los sectores industrial y agrícola de la economía. También se suman los préstamos del Banco de Brasil a las empresas estatales;

d) Redescuentos: también es una cuenta que incluye varios tipos de operaciones. Se registran todos los saldos netos acumulados del redescuento por liquidez. Sin embargo, en el caso de Brasil la política de redescuentos se ha usado para orientar el crédito hacia el sector agrícola, 
transacciones cafetaleras, promoción de exportaciones para una política de precios mínimos, etc.;

e) Política de precios de garantía: el Ministerio de Agricultura está encargado de fijar los precios de garantía para los productos agrícolas. El manejo de fondos necesarios para la compra del exceso de oferta de productos agrícolas, se efectúa a través del Banco de Brasil, y los precios topes para arreglar las fuentes de estas operaciones las propone el Ministerio de Agricultura aprobadas por el Consejo Monetario;

f) Transacciones cafetaleras: la negociación de estas operaciones está bajo la completa responsabilidad del Ministerio de Industria y Comercio (dentro del cual se encuentra el Instituto Brasileño del Café, IBC). Los ingresos que provienen de esta cuenta se derivan de la cuota de retención (algo así como un impuesto en el café administrado por el Consejo Monetario Nacional) y también de las ventas del café de las existencias en el exterior del IBC, y los gastos se originan por làs compras del exceso de oferta, y políticas de redescuentos en las transacciones cafetaleras;

g) Reservas netas de divisas: esta cuenta muestra el valor del cruceiro en las compras netas (o ventas netas) de moneda extranjera debido al superávit o déficit en la balanza de pagos. Esta posición no se fija sólo por el monto del déficit (o superávit) en moneda extranjera, șino también por el tipo de cambio corriente.

Existe todo un grupo de cuentas que no se incluye en este análisis. El Consejo Monetario Nacional puede dar algunos adelantos al Banco Nacional de Desarrollo, a la Caja Económica Federal y a otras muchas agencias financieras del gobierno para cubrir los fondos de ciertas operaciones. Por último, debe reconocerse que el Consejo Monetario Nacional puede captar recursos de cualquier institución que pertenezca al sistema financiero nacional, que eventualmente encaren un excedente de recursos sobre inversiones o, por el contrario, pueden proporcionar recursos a cada uno de ellos. El instrumento que se usa para este propósito son los bonos indizados, desde luego dependiendo de la sola decisión del Consejo Monetario Nacional. Trato de recalcar la flexibilidad que se construye dentro del sistema financiero, mostrando que existe cierto grado de libertad, no sólo para controlar la oferta monetaria total, sino también para trasladar los recursos de una agencia financiera a otra, o para prevenir el impacto expansionario para cada partida del activo en la tasa de crecimiento de monedas fuertes.

Hasta 1964, los elementos dominantes en el crecimiento de monedas fuertes fue el déficit fiscal y los préstamos del Banco de Brasil al sector privado. Esto muestra que probablemente el gobierno estaba usando financiamiento inflacionario como forma para obtener recursos. Puede observarse que además del hecho de que los bonos de reserva a corto plazo se vendían al público, su efecto contraccionista era relativamente pequeño. La eventual discordancia entre el Banco de Brasil y el suMOC 
(Superintendencia de Fondos y Créditos, que fue un sustituto incompleto institucional del Banco Central hasta 1964), podría atribuirse a la excesiva expansión de los préstamos del Banco de Brasil al sector privado. Probablemente este era un elemento muy importante que explicaba que la expansión de la moneda fuerte estaba casi fuera de control en ese período.

Después de 1964, la presión de la tesorería se va reduciendo y al final del período de análisis ésta es la única responsable casi de la quinta parte del crecimiento total. En segundo lugar, las autoridades monetarias comienzan a utilizar la venta de bonos al público como forma para controlar la expansión de la moneda fuerte.

El impacto expansionario del Banco de Brasil nunca disminuyó. Después de 1968, puede observarse que comienza a entrar gran cantidad de moneda extranjera para dominar la expansión de la moneda fuerte. Las entradas y salidas de moneda extranjera crearon cierto tipo de política monetaria, avances paulatinos hasta 1968, que fue cuando se introdujo el sistema político de fijación de tipos de cambio con ajustes graduales (crawling peg). Entre 1964 y los primeros meses de 1968, cuando se ajustó el tipo de cambio, las especulaciones en el movimiento de capital en la balanza de pagos crearon, por algún tiempo, agudas expansiones de la oferta monetaria como la de 1965 y en algunas ocasiones, ciertas reducciones considerables como la de 1966. Se reconoce que el tipo de cambio ajustado en un medio inflacionario provoca esta clase de entradas y salidas en las reservas, y probablemente la baja súbita en la tasa de expansión de la oferta monetaria en 1966, que puede atribuirse a la recesión que ocurrió durante los primeros meses de 1967, causada casi en su totalidad por las salidas de divisas. Es cierto que en 1966 el gobierno tuvo una tasa "objetivo" de inflación de alrededor del $10 \%$ y que trataron de disminuír el aumento de precios por una considerable reducción de la expansión monetaria. Esto se realizó a través de la reducción de la presión fiscal sobre la reserva y a un aumento de ventas de los bonos indizados en el mercado de operaciones abiertas y también a causa de la gran salida de divisas netas; durante los primeros meses de 1967 , la economía ya estaba en una gran depresión. Las autoridades monetarias aumentaron la tasa de expansión de la oferta monetaria, principalmente a través del aumento en el déficit fiscal y también a cierta liberación en los préstamos del Banco de Brasil al sector privado, para poner de esta forma la liquidez real de la economía en el nivel deseado. Desde 1968 puede verse, primero, que el impacto expansionario de la reserva continua descendiendo; en segundo lugar, que el efecto expansionario de los préstamos del Banco de Brasil al sector privado se mantienen más o menos bajo estricto control; que después de la introducción del sistema crawling peg combinado con el mantenimiento de una tasa de interés doméstica por abajo del nivel de la tasa internacional, existe un control de la entrada de divisas, nulificado en gran parte por la venta de bonos indizados al público. 
Quisiera hacer resaltar dos problemas que se analizarán en las dos secciones siguientes: primero, el aumento de la participación de bonos indizados en el total de las monedas fuertes, con lo que el gobierno está devolviendo al público una parte de los ingresos por inflación. Este fenómeno actúa como elemento que inhibe el uso de una tasa inflacionaria por parte del gobierno como forma para movilizar más recursos. En segundo lugar, y debido al hecho de que la mayoría de entradas netas de capital extranjero se presentaba bajo la forma de préstamos canalizados a través del sistema bancario, se mostrará que la tasa de préstamos respecto al total de la oferta monetaria aumentó a partir de 1968. En otro documento se desarrolla la teoría que demuestra que el crédito, en términos reales, al sector privado es un factor muy importante que afecta la oferta agregada de la economía. ${ }^{9}$

En este sentido, no sólo el gobierno trató de reducir la tasa de expansión de la oferta monetaria, para hacer consistente su objetivo de reducir gradualmente la inflación, también trató de cambiar la tasa de préstamo en efectivo para provocar efectos expansionarios en la oferta agregada de la economía.

Desde mi punto de vista, este fue uno de los puntos claves de la política de estabilización brasileña, que se unió a la generalización del índice permitido para alcanzar la reducción gradual de la inflación, tratando de minimizar el costo social.

\section{INGRESOS DEL GOBIERNO PROVENIENTES DE LA INFLACIÓN}

Después de 1964, por un largo período, el gobierno brasileño hizo uso masivo del impuesto inflacionario como forma de movilizar recursos. La utilización de sólo un instrumento (poltica monetaria) para conseguir dos objetivos (estabilización y aumento del ahorro público) tiene una inconsistencia obvia que sólo puede eliminarse si la política monetaria se aplica a la estabilización interna (desde luego se supone una economía "cerrada"), permitiendo de esta forma que la política fiscal y el mercado financiero se hicieran cargo del ahorro.

En esta sección se quieren aclarar tres puntos importantes: a) debido a los retrasos en la respuesta de $\pi$ en relación a los cambios de $\mu$, el ingreso del gobierno por inflación convergerá rápidamente hacia el nuevo equilibrio, persuadiendo al gobierno a usar este mecanismo como instrumento adicional para movilizar recursos; $b$ ) cuando se toma la decisión para la estabilización, el acercamiento gradual mostrará que es la mejor medida para evitar así pérdidas masivas del impuesto inflacionario en el muy corto plazo; c) el hecho de que la participación de los bonos indizados en el incremento de monedas fuertes aumente, redistribuirá al público una parte sustancial del impuesto inflacionario, al reducir los incentivos para utilizar esta forma de financiamiento.

\footnotetext{
- Véase a Pastore y Almonacid [1974].
} 
Cuadro 4

TASA ANUAL DE LA VARIACION DE LA BASE Y SUS COMPONENTES ${ }^{\wedge}$

(Al final del trimestre)

\begin{tabular}{|c|c|c|c|c|c|c|c|c|c|c|}
\hline 1 & & 2 & 3 & 4 & 5 & 6 & 7 & 8 & 9 & 90 \\
\hline 1962 & $\begin{array}{r}\text { II } \\
\text { III } \\
\text { IV }\end{array}$ & $\begin{array}{l}0.451 \\
0.565 \\
0.648 \\
0.626\end{array}$ & $\begin{array}{l}0.317 \\
0.350 \\
0.379 \\
0.391\end{array}$ & $\begin{array}{l}-0.002 \\
-0.002 \\
-0.004 \\
-0.038\end{array}$ & $\begin{array}{l}0.222 \\
0.227 \\
0.238 \\
0.291\end{array}$ & $\begin{array}{l}0.017 \\
0.014 \\
0.010 \\
0.021\end{array}$ & $\begin{array}{l}0.002 \\
0.007 \\
0.008 \\
0.005\end{array}$ & $\begin{array}{r}-0.037 \\
-0.009 \\
0.057 \\
0.029\end{array}$ & $\begin{array}{r}0.078 \\
0.064 \\
-0.008 \\
-0.049\end{array}$ & $\begin{array}{l}-0.156 \\
-0.085 \\
-0.033 \\
-0.025\end{array}$ \\
\hline 1963 & $\begin{array}{r}\text { II } \\
\text { III } \\
\text { IV }\end{array}$ & $\begin{array}{l}0.687 \\
0.677 \\
0.647 \\
0.703\end{array}$ & $\begin{array}{l}0.462 \\
0.432 \\
0.442 \\
0.490\end{array}$ & $\begin{array}{l}-0.039 \\
-0.054 \\
-0.083 \\
-0.056\end{array}$ & $\begin{array}{l}0.286 \\
0.301 \\
0.252 \\
0.210\end{array}$ & $\begin{array}{l}0.050 \\
0.042 \\
0.044 \\
0.015\end{array}$ & $\begin{array}{l}0.003 \\
0.011 \\
0.012 \\
0.011\end{array}$ & $\begin{array}{r}0.024 \\
0.003 \\
-0.075 \\
-0.040\end{array}$ & $\begin{array}{l}-0.003 \\
-0.083 \\
-0.049 \\
-0.015\end{array}$ & $\begin{array}{l}-0.096 \\
0.026 \\
0.103 \\
0.087\end{array}$ \\
\hline 1964 & $\underset{\text { III }}{\text { II }}$ & $\begin{array}{l}0.881 \\
0.977 \\
0.931 \\
0.887\end{array}$ & $\begin{array}{l}0.626 \\
0.677 \\
0.656 \\
0.423\end{array}$ & $\begin{array}{r}-0.061 \\
-0.015 \\
0.042 \\
0.029\end{array}$ & $\begin{array}{l}0.265 \\
0.308 \\
0.314 \\
0.316\end{array}$ & $\begin{array}{l}0.021 \\
0.042 \\
0.032 \\
0.060\end{array}$ & $\begin{array}{c}0.006 \\
0 \\
0.002 \\
0.004\end{array}$ & $\begin{array}{l}-0.038 \\
-0.018 \\
-0.009 \\
-0.076\end{array}$ & $\begin{array}{r}-0.040 \\
-0.012 \\
0.057 \\
0.132\end{array}$ & $\begin{array}{r}0.102 \\
-0.005 \\
-0.162 \\
-0.017\end{array}$ \\
\hline 1965 & $\begin{array}{r}I I \\
\text { III } \\
\text { IV }\end{array}$ & $\begin{array}{l}0.797 \\
0.816 \\
0.819 \\
0.675\end{array}$ & $\begin{array}{l}0.373 \\
0.323 \\
0.244 \\
0.184\end{array}$ & $\begin{array}{r}0.015 \\
-0.016 \\
-0.056 \\
-0.101\end{array}$ & $\begin{array}{l}0.263 \\
0.208 \\
0.151 \\
0.101\end{array}$ & $\begin{array}{r}0.003 \\
-0.015 \\
-0.007 \\
-0.015\end{array}$ & $\begin{array}{l}0.007 \\
0.054 \\
0.108 \\
0.079\end{array}$ & $\begin{array}{l}-0.066 \\
-0.072 \\
0.066 \\
0.042\end{array}$ & $\begin{array}{l}0.214 \\
0.275 \\
0.260 \\
0.212\end{array}$ & $\begin{array}{l}-0.016 \\
0.059 \\
0.183 \\
0.174\end{array}$ \\
\hline 1966 & $\begin{array}{r}\text { I } \\
\text { III } \\
\text { IV }\end{array}$ & $\begin{array}{l}0.600 \\
0.458 \\
0.300 \\
0.216\end{array}$ & $\begin{array}{l}0.136 \\
0.101 \\
0.115 \\
0.106\end{array}$ & $\begin{array}{l}-0.124 \\
=0.154 \\
-0.131 \\
-0.094\end{array}$ & $\begin{array}{l}0.099 \\
0.126 \\
0.121 \\
0.155\end{array}$ & $\begin{array}{l}0.016 \\
0.033 \\
0.041 \\
0.031\end{array}$ & $\begin{array}{r}0.064 \\
0.035 \\
0.013 \\
-0.001\end{array}$ & $\begin{array}{r}0.039 \\
-0.009 \\
-0.028 \\
-0.066\end{array}$ & $\begin{array}{l}-0.008 \\
-0.063 \\
-0.084 \\
-0.113\end{array}$ & $\begin{array}{l}0.380 \\
0.368 \\
0.253 \\
0.198\end{array}$ \\
\hline 1967 & $\begin{array}{r}\text { II } \\
\text { III } \\
\text { IV }\end{array}$ & $\begin{array}{l}0.274 \\
0.279 \\
0.312 \\
0.264\end{array}$ & $\begin{array}{l}0.194 \\
0.241 \\
0.216 \\
0.198\end{array}$ & $\begin{array}{l}-0.124 \\
-0.088 \\
-0.132 \\
-0.117\end{array}$ & $\begin{array}{l}0.142 \\
0.121 \\
0.145 \\
0.149\end{array}$ & $\begin{array}{r}0.002 \\
-0.021 \\
-0.003 \\
0.002\end{array}$ & $\begin{array}{l}0.005 \\
0.007 \\
0.004 \\
0.007\end{array}$ & $\begin{array}{r}-0.069 \\
-0.060 \\
-0.018 \\
0.007\end{array}$ & $\begin{array}{l}-0.012 \\
-0.061 \\
-0.080 \\
-0.082\end{array}$ & $\begin{array}{l}0.147 \\
0.139 \\
0.179 \\
0.099\end{array}$ \\
\hline 1968 & $\begin{array}{r}I \\
\text { II } \\
\text { III } \\
I V\end{array}$ & $\begin{array}{l}0.360 \\
0.340 \\
0.307 \\
0.435\end{array}$ & $\begin{array}{l}0.214 \\
0.155 \\
0.140 \\
0.149\end{array}$ & $\begin{array}{r}-0.064 \\
-0.026 \\
0.017 \\
-0.018\end{array}$ & $\begin{array}{l}0.186 \\
0.224 \\
0.249 \\
0.260\end{array}$ & $\begin{array}{l}0.016 \\
0.021 \\
0.039 \\
0.050\end{array}$ & $\begin{array}{l}0.008 \\
0.009 \\
0.015 \\
0.014\end{array}$ & $\begin{array}{l}-0.014 \\
-0.012 \\
-0.035 \\
-0.072\end{array}$ & $\begin{array}{r}-0.027 \\
-0.022 \\
0.013 \\
0.024\end{array}$ & $\begin{array}{r}0.041 \\
-0.038 \\
-0.131 \\
0.029\end{array}$ \\
\hline 1969 & $\begin{array}{r}\text { I } \\
\text { II } \\
\text { III } \\
\text { IV }\end{array}$ & $\begin{array}{l}0.264 \\
0.262 \\
0.258 \\
0.267\end{array}$ & $\begin{array}{l}0.056 \\
0.061 \\
0.017 \\
0.064\end{array}$ & $\begin{array}{l}-0.017 \\
-0.119 \\
-0.183 \\
-0.151\end{array}$ & $\begin{array}{l}0.210 \\
0.215 \\
0.200 \\
0.208\end{array}$ & $\begin{array}{l}0.062 \\
0.051 \\
0.036 \\
0.011\end{array}$ & $\begin{array}{r}0.011 \\
0.004 \\
0.063 \\
-0.001\end{array}$ & $\begin{array}{l}-0.089 \\
-0.090 \\
-0.060 \\
-0.054\end{array}$ & $\begin{array}{l}0.001 \\
0.065 \\
0.109 \\
0.141\end{array}$ & $\begin{array}{l}0.029 \\
0.076 \\
0.136 \\
0.050\end{array}$ \\
\hline 1970 & $\begin{array}{r}\text { II } \\
\text { III } \\
\text { IV }\end{array}$ & $\begin{array}{l}0.208 \\
0.249 \\
0.212 \\
0.183\end{array}$ & $\begin{array}{l}0.035 \\
0.051 \\
0.050 \\
0.055\end{array}$ & $\begin{array}{l}0.227 \\
-0.187 \\
-0.054 \\
-0.133\end{array}$ & $\begin{array}{l}0.226 \\
0.234 \\
0.207 \\
0.196\end{array}$ & $\begin{array}{l}0.023 \\
0.004 \\
0.018 \\
0.014\end{array}$ & $\begin{array}{r}-0.006 \\
0.022 \\
0.030 \\
0.022\end{array}$ & $\begin{array}{c}0.046 \\
0 \\
-0.093 \\
-0.074\end{array}$ & $\begin{array}{l}0.199 \\
0.198 \\
0.135 \\
0.110\end{array}$ & $\begin{array}{l}0.004 \\
0.072 \\
0.018 \\
0.006\end{array}$ \\
\hline
\end{tabular}

" Los valores que aparecen en las columnas corresponden a:

1. Períodos

2. Base

3. Déficit de la reserva

4. Operaciones de "mercado abierto"

5. Préstamos del Banco de Brasil al sector privado

6. Redescuentos

7. Política de precios mínimos

8. Cuenta del café

9. Reservas extranjeras netas

10. Otras cuentas (saldo neto) 
Si no existiera el sistema bancario y el gobierno central dispusiera del monopolio de creación de moneda, se captarán todos los ingresos del gobierno proveniente de lá inflación mediante:

$$
R=\frac{1}{P} \frac{d M}{d t}=\left(\frac{M}{P}\right) \frac{1}{M} \frac{d M}{d t}
$$

En equilibrio pleno $\left(\operatorname{con} \frac{1}{P}=\left(\frac{M}{P}\right)^{d} ; \pi=\mu=E\right.$ ), y el ingreso real constante, el ingreso sería

$$
R=\left(\frac{1}{P} \frac{d P}{d t}\right)\left(\frac{M}{P}\right)^{d}
$$

que es producto de la "tasa de impuesto" $1 / \mathrm{P} \mathrm{dP} / \mathrm{dt}$, por el "impuesto base" $(\mathrm{M} / \mathrm{P})^{d}$.

Cuando existe un sistema bancario privado, el ingreso del gobierno es:

$$
R_{G}=\frac{1}{K} R
$$

donde $K$ es el multiplicador monetario, y los bancos captarían:

$$
R_{B}=\frac{K-1}{K} R
$$

si se pueden ajustar las tasas de interés nominal sobre los préstamos a la tasa de inflación esperada.

Si se supone un valor del multiplicador monetario alrededor de 2 , el gobierno captaría el 50\% del ingreso total. El cuadro 5 muestra los niveles de este ingreso como proporción del ingreso real (al valor medio

\begin{tabular}{|c|c|c|c|}
\hline 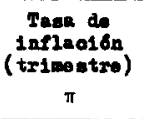 & $\begin{array}{l}\text { Saldo on } \\
\text { of eotivo } \\
\text { real } \\
x^{d}\end{array}$ & $\begin{array}{l}\text { Ingrasos } \\
\mathbf{n}^{\mathrm{d}}\end{array}$ & $B / Y$ \\
\hline $\begin{array}{l}0.05 \\
0.10 \\
0.20 \\
0.30 \\
0.40 \\
0.50 \\
0.60 \\
0.70 \\
0.80\end{array}$ & $\begin{array}{r}208.7 \\
193.6 \\
166.5 \\
143.3 \\
123.2 \\
106.2 \\
93.6 \\
78.6 \\
67.7\end{array}$ & $\begin{array}{l}10.4 \\
19.4 \\
33.3 \\
43.0 \\
49.3 \\
53.1 \\
56.2 \\
55.0 \\
54.2\end{array}$ & $\begin{array}{l}0.04 \\
0.07 \\
0.11 \\
0.14 \\
0.16 \\
0.18 \\
0.19 \\
0.18 \\
0.18\end{array}$ \\
\hline
\end{tabular}
trimestral de 1970), para disintas tasas de inflación en equilibrio pleno.

Cuadro 5

INGRESOS DEL GOBIERNO PROVENIENTES DE LA INFLACIÓN 
El ingreso para las tasas de inflación entre $40 \%$ y $50 \%$ anuales, sería alrededor del $10 \%$ del ingreso real.

Sin embargo , un ejercicio más interesante es apreciar cómo converge el ingreso a un nuevo equilibrio, cuando las autoridades monetarias deciden efectuar un cambio en $\mu$ de una vez por todas. La trayectoria de $\pi$ estimada en la sección III permite derivar los datos incluidos en los cuadros 6 y 7 .

En el primer cuadro, se supone que $\mu=\pi$ al $5 \%$ trimestral y las autoridades monetarias incrementan $\mu$ al $10 \%$ trimestral. El cociente $R / y$, que era del $4 \%$, será del $8 \%$ en una situación de equilibrio pleno. Sin embargo, si se incrementara $\mu$ en el trimestre $R / y$ subiría a $8.5 \%$ y después oscilaría hasta converger al nuevo punto de equilibrio. Esto muestra que el gobierno obtendría inmediatamente un ingreso más alto al de la posición de nuevo equilibrio.

El cuadro 6 muestra el ejercicio opuesto. Suponiendo que $\mu=\pi=$ 0.20 por trimestre y que $\mu$ se redujera al $5 \%$. El cociente $R / y$, que era de $13 \%$ declinará inmediatamente a $3 \%$, y luego oscilará hasta que alcance el nuevo equilibrio al nivel de $R / y=0.04$.

La crisis monetaria implicará una reducción sustancial en los gastos públicos y si los costos son decrecientes de manera inflexible, afectará directamente a la inversión pública al reducir la tasa de crecimiento. El sistema bancario obtendría una parte de este ingreso que crecerá si $\pi$ aumenta (hasta el punto donde la elasticidad-costo de la demanda de fondos fuera 1). Pero una crísis monetaria afectaría sus ingresos $y$, dependiendo de su estructura de costos, probablemente algunos se irían a la bancarrota.

Pienso que estos argumentos son importantes para formular el caso de "aproximaciones sucesivas" en vez de una "crisis monetaria".

Por último, como está aumentando la participación de los bonos indizados en el activo total de las autoridades monetarias, el gobierno redistribuye al público una parte de su ingreso proveniente de la inflación, creando así una trampa que convierte al impuesto inflacionario en su propio destructor.

Cuadro 6

INGRESOS DEL GOBIERNO PROVENIENTES DE LA INFLACIÓN

\begin{tabular}{|c|c|c|c|c|c|c|}
\hline & $\mu$ & $\pi$ & $\mu-\pi$ & $\begin{array}{r}m / p \\
\text { (roal) }\end{array}$ & $\mathrm{B}$ & $\mathrm{R} / \mathrm{s}$ \\
\hline Equilibrio intolal & $\begin{array}{l}0.05 \\
0.05 \\
0.10 \\
0.10 \\
0.10 \\
0.10\end{array}$ & $\begin{array}{l}0.050 \\
0.050 \\
0.066 \\
0.082 \\
0.097 \\
0.111\end{array}$ & $\begin{array}{c}0 \\
0 \\
0.034 \\
0.018 \\
0.003 \\
-0.011\end{array}$ & $\begin{array}{l}160.6 \\
160.6 \\
166.1 \\
66.0 \\
169.6 \\
167.7\end{array}$ & $\begin{array}{r}8.03 \\
8.03 \\
16.60 \\
16.91 \\
16.96 \\
16.77\end{array}$ & $\begin{array}{l}0.040 \\
0.040 \\
0.085 \\
0.086 \\
0.087 \\
0.086\end{array}$ \\
\hline Equilibrio finel & 0.10 & 0.100 & 0 & 149.4 & 14.90 & 0.080 \\
\hline
\end{tabular}


VIII. MODElo EXPliCATIVo DEL SALDO NOMINAL DE PRÉSTAMOS
BANCARIOS

Antes que nada, es evidente que un aumento en la base monetaria debido al aumento en alguna de las partidas del activo de las autoridades monetarias, causará (los coeficientes multiplicadores constantes) una elevación de los préstamos bancarios debido al impacto que produce sobre la oferta monetaria total y principalmente sobre los depósitos bancarios. Este incremento de los préstamos bancarios nominales no depende del canal por el que se ha aumentado la base monetaria y básicamento se debe al aumento de la existencia monetaria.

Si la base monetaria aumenta debido al incremento en el monto de los préstamos del Banco de Brasil al sector privado, tendrá lugar un aumento en los dos efectos. El primero corresponde al efecto indirecto, similar al que se explicó antes como activo debido al aumento de la base monetaria; el segundo corresponde, al efecto directo para hacer aumentar la base en sí a través de préstamos del Banco de Brasil al sector privado.

De la misma forma, si se efectúa un aumento en la base monetaria, ya sea porque las autoridades monetarias hayan aumentado el límite superior del redescuento comercial bancario, o porque hayan dejado entrar al país reservas netas como resultado de operaciones financieras entre brasileños y bancos extranjeros, para efectos de préstamos al sector privado, entonces tendrá lugar el citado aumento en estos dos efectos, el directo y el indirecto.

Las situaciones descritas en los dos párrafos anteriores, originan impactos diferentes: por ejemplo, al causar un incremento en la base debido al déficit fiscal. En este caso aumentarán los préstamos originados por el incremento de la oferta monetaria inducida por el crecimiento de la base.

Para desarrollar el modelo, hay que definir el total de préstamos bancarios en términos nominales:

$$
L:=L^{B B}+L^{B C}
$$

donde $L^{B C}$ representa el saldo nominal de los préstamos bancarios comerciales y $L^{B B}$ representa el saldo nominal de los préstamos del Banco de Brasil.

Desde luego que los bancos comerciales podrían expandir sus préstamos hasta el límite de un cociente $(1-r)$ del total de depósitos, al cual se necesitaría añadir:

a) el redescuento total, $\mathrm{RD}$;

b) el saldo de los propios recursos de los bancos que la banca comercial no quiere aplicar a otras partidas que no sean préstamos, $K$;

c) la parte correspondiente a los recursos financieros extranjeros convertidos a cruceiros que entran al país a través de bancos comerciales, 
$\alpha F_{r}$, donde $F_{r}$ representa el total de la "balanza de pagos", de los que se obtiene: ${ }^{10}$

$$
L^{B C}:=(1-r) D^{B C}+R D+\alpha F_{r}=K
$$

El redescuento se adopta como un recurso del banco en el momento que, debido a una situación anormal por falta de efectivo, se ve obli- gado a recurrir al Banco Central. Sin embargo, las autoridades monetarias han permitido el uso de este instrumento con alguna flexibilidad, creando así áreass especiales con costos más bajos, deseando orientar el crédito hacia algunos sectores como la agricultura, café, etc.

Las autoridades monetarias permiten a los bancos efectuar operaciones en el extranjero adquiriendo recursos en divisas que se venden al Banco Central para obtener cruceiros a cambio. Desde luego que un superávit en la balanza de pagos origina un incremento en la base monetaria. Pero se necesita diferenciar del total de las reservas extranjeras netas aquellas operaciones financieras que efectúan los bancos brasileños con los bancos extranjeros, para obtener recursos con los que aumentan el monto disponible de cruceiros, para incrementar los préstamos directos al sector privado. Este es el caso típico de operaciones como la "Resolución 63" del Banco Central, la que en su mayoría es causa de expansión en la base, pero al mismo tiempo también origina un aumento directo en los préstamos al sector privado. Supóngase que $F_{r}$ representa el total de recursos en moneda extranjera que ha entrado al país, y que una proporción $\alpha$ de estos recursos entra a través de bancos comerciales, lo que significa un aumento directo de préstamos. Si se usa la expresión del multiplicador monetario se llega a:

$$
L^{B C}=(\delta M)+R D+\alpha F_{R}+K
$$

Se puede tomar el total de aplicaciones $B$, separando las operaciones que no crean préstamos directos y escribir la base:

$$
B=C_{g}+L^{B B}+R D+\alpha F_{r}+(1-\alpha) F_{r}
$$

donde todos los símbolos han sido definidos. En forma simplificada:

$$
B_{2}=C_{g}+(1-\alpha) F_{r} \text { y } B_{1}=L^{B B}+R D+F_{r}
$$

\footnotetext{
10 Parte de los redescuentos, de la intermediación en la entrada de reservas extranjeras y de los propios recursos de los bancos, pueden aparecer como efectivo, y debe multiplicarse el segundo término del lado derecho de $(8.2)$ por $\left(1-r^{\prime}\right)$ donde $r^{\prime}$ es la "tasa de reserva" de estas partidas. Por lo tanto, se piensa que $r^{\prime}$ es muy pequeña y que no afecta el análisis si se supone que es aproximadamente cero.
} 
Al multiplicar ambos lados por el multiplicador monetario, se obtiene:

$$
L^{B B}=(1-\delta) M-B_{2}
$$

donde $\delta$ son filtraciones del multiplicador monetario.

La ecuación (8.5) produce una forma para planear, dentro de un presupuesto financiero, la política de préstamos del Banco de Brasil. Supóngase que las autoridades monetarias tienen una estimación acerca de la entrada de reservas extranjeras netas. Si quieren aumentar los préstamos del Banco de Brasil al sector privado, aún tienen que reducir $B_{1}$ que significa vender valores al sector privado para reducir el déficit en el presupuesto $\mathrm{y}$ algunas otras hipótesis alternativas.

Para encontrar el total de la oferta de préstamos bancarios en términos nominales, sólo hay que substituir (8.3) y (8.5) en (8.1) para obtener

$$
L=M\left[1-(1-\delta) \frac{B_{2}}{B}\right]
$$

Si se suponen constantes los coeficientes multiplicadores, dado que $B_{2}$ y $B$ pueden aumentar a tasas diferentes, una vez que las autoridades monetarias pueden cambiar los canales a través de los que inyectan dinero a la economía, se concluye que la tasa de préstamos al total de la oferta monetaria puede ser muy inestable y tener un comportamiento básicamente dependiente sobre la composición de la base monetaria. Esta relación será:

$$
\frac{L}{M}=1-(1-\delta) \frac{B_{2}}{B}
$$

que depende de la composición de los requerimientos de las autoridades monetarias. Si se supone una situación de equilibrio en la que las tasas de crecimiento monetarias son iguales a la tasa de aumento de los precios, por lo que se mantiene constante la oferta monetaria real, el sistema bancario real de préstamos podría aumentar si la tasa de incremento de $B_{2}$ es mayor que la tasa de crecimiento de $B$. El control de la composición de la base monetaria, y su nivel, garantizan también el control no sólo de la misma expansión monetaria, sino también del cociente entre los préstamos bancarios y los medios de pago.

El análisis del cuadro 4 arroja algunas indicaciones importantes. Antes que nada, cuando en 1965 el gobierno intentó efectuar una reducción más alta de la tasa de expansión monetaria (interrumpida por 
la entrada de reservas líquidas extranjeras), se efectuó una marcada reducción de la expansión de las tasas de préstamos del Banco de Brasil al sector privado.

En 1965, el cociente de prástamos alcanzó su punto mínimo mostrando que en el intento de controlar la inflación por medio de políticas monetarias, las autoridades monetarias terminaron por originar un descenso en la oferta real de préstamos en relación al nivel que pudo haber alcanzado.

En períodos recientes, la expansión base ha sido controlada por una marcada reducción en el impacto expansionario del déficit en el presupuesto y por operaciones de mercado abierto. Los elementos principales en la expansión base han sido los préstamos del Banco de Brasil al sector privado y en las reservas netas extranjeras, ambos para aumentar los préstamos bancarios.

Para valorar el impacto originado por las reservas extranjeras sobre los préstamos ordinarios de los bancos comerciales, se usará $\left(L^{*}\right)^{\mathrm{BC}}$ como el máximo volumen que tales bancos podrían prestar si no contaran con las operaciones financieras en moneda extranjera; o sea:

$$
\left(L^{*}\right)^{B C}=(1-r) D^{B C}+R D
$$

Con el inicio de la intermediación regulada por la "Instrucción 63" del Banco Central, estos recursos aumentaron y los bancos comerciales pudieron incrementar sus préstamos en relación con sus niveles anteriores. Si se admite que la proporción de las reservas extranjeras netas que entran al país, explican el exceso real del préstamo sobre el máximo prestable potencialmente, se obtiene:

$$
L^{B C}-\left(L^{*}\right)^{B C}=\alpha F_{r}
$$

La gráfica 4 y el cuadro 5 muestran una comparación entre el exceso de préstamos actuales sobre el máximo prestable potencialmente y el

Cuadro 7

INGRESOS DEL GOBIERNO PROVENIENTES DE LA INFLACIÓN

\begin{tabular}{lcccccc}
\hline & $\mu$ & $\pi$ & $\mu-\pi$ & $\begin{array}{c}\text { N/P } \\
\text { (real) }\end{array}$ & $\frac{M}{\mathrm{P}} \pi=\mathrm{R}$ & $\mathrm{H} / \mathrm{J}$ \\
\hline Equilibrio 1niotal & 0.20 & 0.20 & 0 & 128.5 & 25.7 & 0.13 \\
& 0.20 & 0.20 & 0 & 128.5 & 25.7 & 0.13 \\
& 0.05 & 0.15 & -0.10 & 116.8 & 5.8 & 0.03 \\
& 0.05 & 0.10 & -0.05 & 111.2 & 5.6 & 0.03 \\
& 0.05 & 0.06 & -0.01 & 110.1 & 5.5 & 0.03 \\
Equilibrio final & 0.05 & 0.03 & 0.02 & 112.3 & 5.6 & 0.03 \\
\hline
\end{tabular}


PASTORE: POLITICA MONETARIA DE BRASIL

Gráfica 6

EXPANSIÓN DE PRÉSTAMOS BANCARIOS Y RESERVAS

EXTRANJERAS NETAS

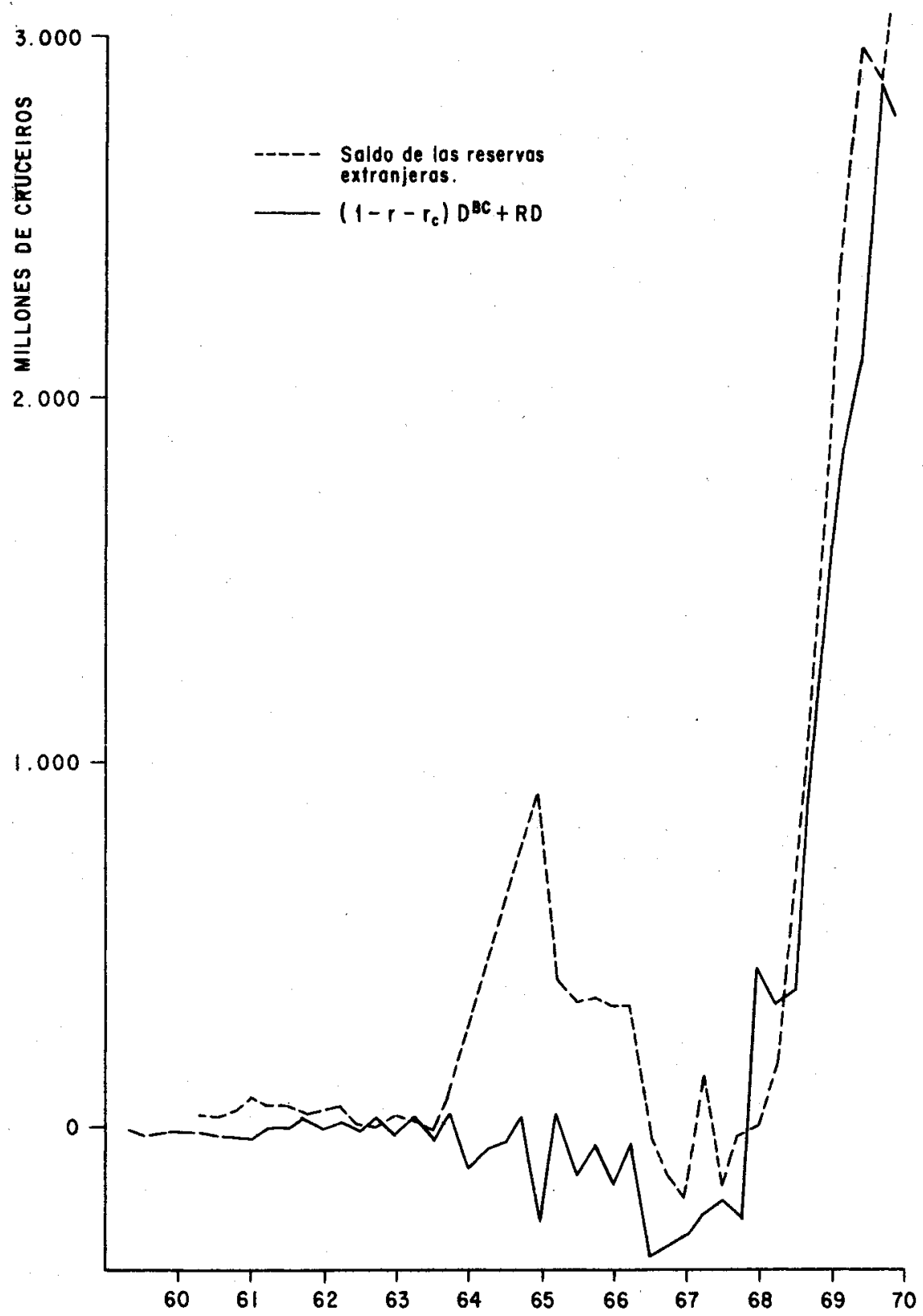


Cuadro 8

ACERVo de RESERVAS EXTRANJERAS Y PRÉSTAMOS BANCARIOS ${ }^{a}$

\begin{tabular}{|c|c|c|c|c|c|}
\hline 1 & $\stackrel{2}{(A)}^{2}$ & (B) & 4 & 5 & 6 \\
\hline $\begin{array}{ll}1960 \quad \text { I } \\
& \text { II } \\
& \text { III } \\
& \text { IV }\end{array}$ & $\begin{array}{l}287.4 \\
308.4 \\
340.7 \\
332.4\end{array}$ & $\begin{array}{l}303.6 \\
331.0 \\
356.9 \\
397.2\end{array}$ & $\begin{array}{l}-16.2 \\
=22.7 \\
=16.2 \\
-14.8\end{array}$ & $\begin{array}{r}96 \\
130 \\
139 \\
141\end{array}$ & \\
\hline $1961 \begin{array}{r}\text { I } \\
\text { II } \\
\text { III } \\
\text { IV }\end{array}$ & $\begin{array}{l}394.3 \\
410.1 \\
444.7 \\
501.7\end{array}$ & $\begin{array}{l}413.9 \\
436.9 \\
472.2 \\
533.0\end{array}$ & $\begin{array}{l}-14.6 \\
=26.6 \\
=27.5 \\
-31.4\end{array}$ & $\begin{array}{l}123 \\
125 \\
171 \\
278\end{array}$ & $\begin{array}{l}27 \\
27 \\
41 \\
74\end{array}$ \\
\hline $1962 \begin{array}{r}\text { I } \\
\text { II } \\
\text { III } \\
\text { IV }\end{array}$ & $\begin{array}{l}561.6 \\
593.6 \\
681.6 \\
775.0\end{array}$ & $\begin{array}{l}564.6 \\
596.9 \\
656.6 \\
780.6\end{array}$ & $\begin{array}{r}-3.0 \\
-\quad 3.2 \\
25.0 \\
-\quad 5.6\end{array}$ & $\begin{array}{l}231 \\
219 \\
181 \\
192\end{array}$ & $\begin{array}{l}59 \\
54 \\
37 \\
44\end{array}$ \\
\hline $\begin{array}{lr}1963 & I \\
& \text { II } \\
& \text { III } \\
& \text { IV }\end{array}$ & $\begin{array}{r}807.7 \\
052.1 \\
968.9 \\
1209.9\end{array}$ & $\begin{array}{r}797.6 \\
363.8 \\
936.5 \\
1235.0\end{array}$ & $\begin{array}{r}10.2 \\
-11.7 \\
-32.4 \\
-25.0\end{array}$ & $\begin{array}{l}215 \\
123 \\
120 \\
163\end{array}$ & $\begin{array}{r}57 \\
-\quad 1 \\
-\quad 2 \\
29\end{array}$ \\
\hline $\begin{array}{l}1964 \quad \text { I } \\
\text { II } \\
\text { III } \\
\text { IV }\end{array}$ & $\begin{array}{ll}1 & 367.6 \\
1 & 595.4 \\
1 & 951.6 \\
2 & 213.9\end{array}$ & $\begin{array}{ll}1 & 336.4 \\
1 & 633.3 \\
1 & 909.7 \\
2 & 333.0\end{array}$ & $\begin{array}{r}31.2 \\
-38.0 \\
41.9 \\
-114.1\end{array}$ & $\begin{array}{l}147 \\
121 \\
175 \\
276\end{array}$ & $\begin{array}{r}17 \\
-\quad 14 \\
73 \\
253\end{array}$ \\
\hline $\begin{array}{ll}1965 & \text { I } \\
& \text { II } \\
\text { III } \\
\text { IV }\end{array}$ & $\begin{array}{ll}2 & 3.7 .1 \\
2 & 3.40 .4 \\
3 & 430.3 \\
3 & 939.1\end{array}$ & $\begin{array}{ll}2 & 452.0 \\
2 & 905.4 \\
3 & 398.1 \\
4 & 222.8\end{array}$ & $\begin{array}{r}-64.9 \\
57.0 \\
32.2 \\
-283.7\end{array}$ & $\begin{array}{l}367 \\
453 \\
538 \\
625\end{array}$ & $\begin{array}{l}421 \\
589 \\
737 \\
930\end{array}$ \\
\hline $1966 \begin{array}{r}\text { I } \\
\text { II } \\
\text { III } \\
\text { IV }\end{array}$ & $\begin{array}{l}3953.6 \\
4221.3 \\
4519.2 \\
4395.0\end{array}$ & $\begin{array}{ll}3 & 920.7 \\
4 & 360.7 \\
4 & 567.2 \\
5 & 058.0\end{array}$ & $\begin{array}{r}24.9 \\
-147.4 \\
-43.0 \\
-163.9\end{array}$ & $\begin{array}{l}383 \\
358 \\
363 \\
352\end{array}$ & $\begin{array}{l}393 \\
337 \\
343 \\
324\end{array}$ \\
\hline $1967 \begin{array}{r}\text { I } \\
\text { II } \\
\text { III } \\
\text { IV }\end{array}$ & $\begin{array}{ll}5 & 007.0 \\
6 & 016.6 \\
6 & 003.2 \\
8 & 950.0\end{array}$ & $\begin{array}{l}5.043 .9 \\
6.371 .8 \\
7.52 .1 \\
8.347 .4\end{array}$ & $\begin{array}{r}-41.9 \\
-355.2 \\
228.9 \\
-295.9\end{array}$ & $\begin{array}{l}343 \\
216 \\
174 \\
146\end{array}$ & 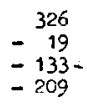 \\
\hline $\begin{array}{ll}1968 \text { I } \\
& \text { II } \\
& \text { III } \\
& \text { IV }\end{array}$ & $\begin{array}{rl}8 & 347.5 \\
16 & 010.1^{1} \\
11 & 404.7+ \\
12 & 813.7\end{array}$ & $\begin{array}{rr}9 & 007.2 \\
10 & 213.2 \\
11 & 705.1 \\
12 & 303.5\end{array}$ & $\begin{array}{r}-230.0 \\
-208.1 \\
-250.4 \\
429.6\end{array}$ & $\begin{array}{l}254 \\
154 \\
194 \\
200\end{array}$ & $\begin{array}{r}139 \\
-103 \\
-\quad 30 \\
-\quad 11\end{array}$ \\
\hline $1969 \begin{array}{r}\text { I } \\
\text { II } \\
\text { III } \\
\text { IV }\end{array}$ & $\begin{array}{l}13387.0 \\
14453.0 \\
15862.0 \\
18114.0\end{array}$ & $\begin{array}{ll}13 & 062.0 \\
14 & 089.0 \\
14 & 826.0 \\
16 & 621.0\end{array}$ & $\begin{array}{ll} & 325.0 \\
364.0 \\
1 & 037.0+ \\
1 & 493.0\end{array}$ & $\begin{array}{l}239 \\
319 \\
148 \\
600\end{array}$ & $\begin{array}{r}145 \\
467 \\
1101 \\
1650\end{array}$ \\
\hline $\begin{array}{ll}1970 & \text { I } \\
& \text { II } \\
& \text { III } \\
& \text { IV }\end{array}$ & $\begin{array}{ll}19 & 141.0 \\
21 & 007.0 \\
22 & 454.0 \\
24 & 287.0\end{array}$ & $\begin{array}{r}17.170 .0 \\
18879.0 \\
19590.0 \\
21537.0\end{array}$ & $\begin{array}{ll}1 & 871.0 \\
2 & 128.0+ \\
2 & 864.0 \\
2 & 750.0\end{array}$ & $\begin{array}{l}794 \\
892 \\
873 \\
962\end{array}$ & $\begin{array}{l}2503 \\
2954 \\
2067 \\
3 \\
3\end{array}$ \\
\hline
\end{tabular}

a Los valores que aparecen en las columnas corresponden a:

1. Período

2. Préstamos de los bancos comerciales

3. $\left(1-r_{v}-r_{c}\right) D^{B C}+R$

4. (A) - (B)

5. Reservas extranjeras netas (en millones de dólares)

6. Reservas extranjeras netas (en millones de cruceiros) 
saldo de la reserva líquida extranjera en cruceiros. Hasta 1964 no se encuentra la presencia de exceso de préstamos de la banca comercial sobre el máximo prestable potencialmente permisible; en el mismo período, la presión expansionaria de las reservas netas extranjeras fue muy reducida.

Durante 1965 se empezó a encontrar algunas perturbaciones. Antes que nada, se originó una reducción en los préstamos reales sobre el máximo prestable potencialmente permisible, probablemente debido a requerimientos alternativos ofrecidos a bancos comerciales. ${ }^{11}$

En segundo lugar, las reservas netas foráneas crecieron marcadamente sin acusar el incremento correspondiente en los préstamos ordinarios de la banca comercial. Lo que ocurrió fue que en 1965 estaba operando la "Instrucción 289" del Banco Central, la que permitió operaciones financieras entre empresas nacionales y extranjeras sin la mediación de los bancos comerciales. Claramente se nota la existencia de una expansión en el crédito al sector privado (o más específicamente, a las empresas con acceso al mercado internacional de capital) que sin embargo no se canalizó a través de bancos comerciales.

En 1968 se observa un cambio en las reservas extranjeras, nuevamente sin nigún efecto visible sobre los préstamos bancarios debido a que estaba todavía en vigor la "Instrucción 289". Indefectiblemente, esto se efectúa con una total contracción del crédito. En 1967, la "Instrucción 289" se restringió gradualmente y las operaciones internacionales financieras comenzaron a ser reguladas mediante la "Instrucción 63" del Banco Central. ${ }^{12}$ De ahí en adelante un banco brasileño podría ser siempre mediador en operaciones para canalizar dinero obtenido por venta de moneda extranjera a las autoridades monetarias.

Resulta fácil observar que con el crecimiento de reservas extranjeras, se registra siempre un crecimiento paralelo en el exceso de préstamos reales sobre el máximo potencialmente prestable, cuando éstas operaciones no estaban presentes. El impacto de este instrumento puede analizarse por el hecho de que al final de este período los recursos alcanzaban ya $10 \%$ del saldo de préstamos de los bancos comerciales al sector privado.

Al combinar los resultados de los cuadros 4 y 5 , se observa que el cambio en los canales de inyección de moneda en la economía fue el punto básico para cambiar la tasa de préstamos de la oferta monetaria.

\footnotetext{
"Al principio de la práctica de indización, hay evidencias de que las autoridades monetarias sobrevaloraron algunas partidas financieras y que resultó más atractivo de lo esperado. Me refiero específicamente a la "Resolución 21" del Banco Central y aunque no hay datos para verificar esta hipótesis, es muy probable que los bancos hayan "huído" de los préstamos, recurriendo voluntariamente a los bonos. Otro mecanismo que pudo haber actuado con el mismo objetivo fue la imposición de efectuar una revalorización de partidas, a lo que se ofreció la alternativa de comprar bonos de tesorería.

${ }^{12}$ La "Instrucción 289" se abolió finalmente en 1969.
} 
En la medida en que el incremento de la oferta de préstamos tuvo alguna importancia para incorporar la política de reducción gradual de la tasa de expansión monetaria (así como de la inflación) y, mientras se apoyara el nivel de crecimiento de la actividad, se concluye que esto era importante para lograr los objetivos fijados antes en el programa de estabilización brasileño.

\section{Oferta de préstamos reales}

De (8.6) se obtiene

$$
\frac{L}{P}=\frac{M}{P}\left[1-(1-\delta) \frac{B_{2}}{B}\right]
$$

que muestra que los préstamos reales del sistema bancario al sector privado dependen del comportamiento del saldo en efectivo real, de los coeficientes del multiplicador monetario y de la composición de la base monetaria.

En un trabajo anterior se mostró que $r$ y $h$ reaccionan muy poco a los cambios en $Y$ y a las tasas de interés, y que el elemento dominante que explica los cambios en $k$ son los requerimientos de reserva obligatoria. ${ }^{13}$ Como $(M / P$ ) es (en el largo plazo) una función estable del ingreso real y el costo de la retención monetaria:

$$
\frac{M}{P}=f(Y, E)
$$

con $f_{y}^{\prime}>0$ y $f_{E}^{\prime}<0$, si $B_{2}$ y $B$ crecen a la misma tasa, los préstamos reales presentarán el mismo comportamiento de los saldos reales en efectivo. Si estamos interesados en una trayectoria de equilibrio de $L / P$ se puede substituir (9.2) por (9.1), recordando que:

$$
E=\pi=\mu-\eta \gamma
$$

donde $\eta=d m^{d} / d y$ y $\gamma=d y / d t$, se obtiene

$$
\frac{L}{P}=f\left\{y_{0} e^{\gamma t} ; \mu-\eta \gamma\right\}\left\{1-(1-\delta) \frac{B_{20} e^{b} t^{t}}{B_{0} e^{b t}}\right\}
$$

donde $b_{2}$ y $b$ son las tasas de crecimiento de $B_{2}$ y $B$.

En la gráfica 7 la curva $L_{0} L_{0}$ muestra la relación entre $L / P$ y $\mu$

1s Véase Pastore [1973]. 
Gráfica 7

OFERTA DE PRÉSTAMOS DE EQUILIBRIO PLENO

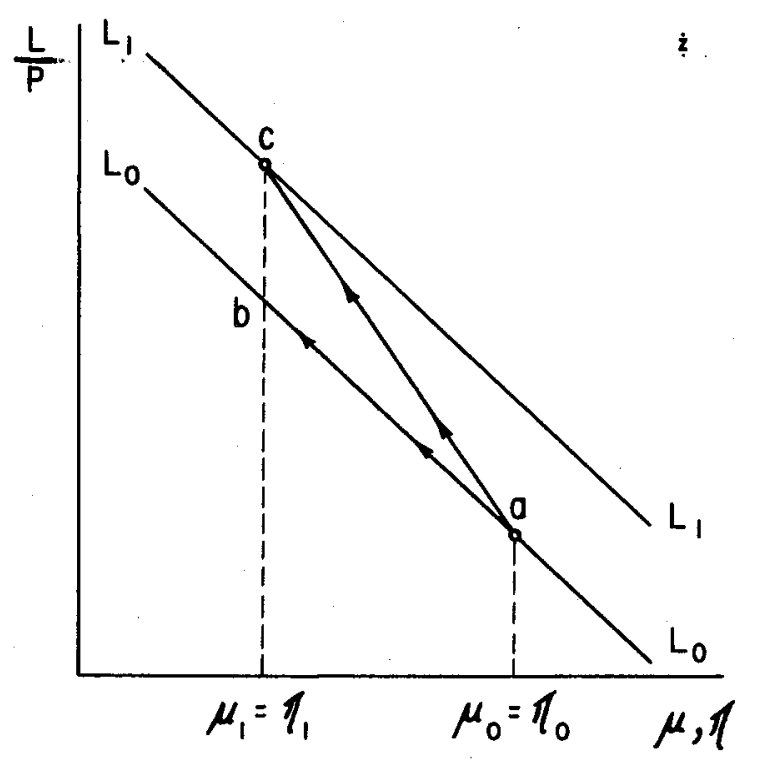

cuando. $b_{2}=b=\mu$ y $y$ es constante. En este caso, la reducción gradual en $\mu$ genera un aumento $L / P$ a lo largo de la curva $L_{0} L_{0}$. Si $b_{2}<b=\mu$, la curva $L L$ irá ascendiendo gradualmente y la trayectoria de crecimiento de $L / P$ llegará eventualmente a una posición entre los puntos $a$ y $c$.

Una crisis monetaria (aun cuando se mantenga la misma composición de la base monetaria) producirá una reducción de préstamos reales al sector privado. Si esta crisis se efectúa a través de una reducción en $B_{1}$ comparada con $B_{2}$, la curva $L L$ girará en forma descendente y los ciclos en $L / P$ serán de más amplitud que en $M / P$.

Si las autoridades monetarias tienen como meta gradualmente la inflación sin fluctuaciones sustanciales en $M / P$ y $L / P$, tendrán que:

a) reducir $\mu$ gradualmente para acercarse lo más posible a la trayecria de equilibrio de $M / P$;

b) cambiar la composición de las partidas desplazando $L L$ a la derecha lo que puede hacerse al reducir las presiones del déficit fiscal, aumentando la participación de $L^{B B}$, redescuentos y en la base monetaria total. 


\section{BIBLIOGRAFIA}

P. Cagan, "The Monetary Dynamics of Hyperinflations", en M. Friedman (Comp.) Studies in the Ouantity Theory of Money, The University of Chicago Press, 1956.

G.C. Chow, "On the Long-Run and Short-Run Demand for Money", Journal of Political Economy, vol. 74, abril de 1966.

P.J. Dhrymes, Distributed Lags: Problems of Estimation and Formulation, Holden Day Inc, 1971.

A.C. Diz, "Money and Prices in Argentina, 1935-1962", en D. Meiselman (Comp.), Varieties of Monetary Experiences, The Chicago University Press, 1970.

S. Goldberg, Introduction to Difference Equations, Nueva York, J. Willey and Sons, 1961.

Z. Griliches, "Distributed Lags: A Survey", Econometrica, vol. 35, enero de 1967.

A.C. Harberger, "The Case of the three Numeraires", trabajo presentado al encuentro anual de la Sociedad Econométrica, diciembre de 1966.

S.A. Morley, "Inflation and Stagnation in Brazil", Economic Development and Cultural Change, enero de 1971.

J.F. Muth, "Optimal Properties of Exponentially Weighted Forecasts", Journal of the American Statistical Associate, vol. 55, 1960.

M. Nerlove, "Estimates of the Elasticities of Supply of Selected Agricultural Commodities", Journal of Farm Economics, vol. 38, 1956.

A.C. Pastore y R.D. Almonacid, "Inflação: Gradualismo ou tratamento de choque. Consideraçôes em torno dos custos da estabilização," mimeografiado, 1974.

A.C. Pastore, "Observações sobre a política monetåria no programa Brasileiro de estabilização", Tesis de docencia, no publicada, São Paulo (1973.a).

A.C. Pastore, "A oferta de moeda no Brasil-1961/72", Pesquisa e Planejamento Eco. nómico, vol. 3, diciembre de (1973.b)

M.H. Simonsen, "Inflação: Gradualismo vs. tratamiento de choque", APEc, Río de Janeiro, 1970. 